\title{
The Vector-Valued Functions Associated with Circular Cones
}

\author{
Jinchuan Zhou ${ }^{1}$ and Jein-Shan Chen ${ }^{2,3}$ \\ ${ }^{1}$ Department of Mathematics, School of Science, Shandong University of Technology, Zibo, Shandong 255049, China \\ ${ }^{2}$ Department of Mathematics, National Taiwan Normal University, Taipei 11677, Taiwan \\ ${ }^{3}$ Mathematics Division, National Center for Theoretical Sciences, Taipei, Taiwan
}

Correspondence should be addressed to Jein-Shan Chen; jschen@math.ntnu.edu.tw

Received 6 April 2014; Accepted 15 May 2014; Published 22 June 2014

Academic Editor: Jen-Chih Yao

Copyright (C) 2014 J. Zhou and J.-S. Chen. This is an open access article distributed under the Creative Commons Attribution License, which permits unrestricted use, distribution, and reproduction in any medium, provided the original work is properly cited.

\begin{abstract}
The circular cone is a pointed closed convex cone having hyperspherical sections orthogonal to its axis of revolution about which the cone is invariant to rotation, which includes second-order cone as a special case when the rotation angle is 45 degrees. Let $\mathscr{L}_{\theta}$ denote the circular cone in $\mathbb{R}^{n}$. For a function $f$ from $\mathbb{R}$ to $\mathbb{R}$, one can define a corresponding vector-valued function $f^{\mathscr{L}_{\theta}}$ on $\mathbb{R}^{n}$ by applying $f$ to the spectral values of the spectral decomposition of $x \in \mathbb{R}^{n}$ with respect to $\mathscr{L}_{\theta}$. In this paper, we study properties that this vector-valued function inherits from $f$, including Hölder continuity, $B$-subdifferentiability, $\rho$-order semismoothness, and positive homogeneity. These results will play crucial role in designing solution methods for optimization problem involved in circular cone constraints.
\end{abstract}

\section{Introduction}

The circular cone is a pointed closed convex cone having hyperspherical sections orthogonal to its axis of revolution about which the cone is invariant to rotation, which includes second-order cone as a special case when the rotation angle is 45 degrees. Let $\mathscr{L}_{\theta}$ denote the circular cone in $\mathbb{R}^{n}$. Then, the $n$-dimensional circular cone $\mathscr{L}_{\theta}$ is expressed as

$$
\mathscr{L}_{\theta}:=\left\{x=\left(x_{1}, x_{2}\right)^{T} \in \mathbb{R} \times \mathbb{R}^{n-1} \mid \cos \theta\|x\| \leq x_{1}\right\} .
$$

The application of $\mathscr{L}_{\theta}$ lies in engineering field, for example, optimal grasping manipulation for multigingered robots; see [1].

In our previous work [2], we have explored some important features about circular cone, such as characterizing its tangent cone, normal cone, and second-order regularity. In particular, the spectral decomposition associated with $\mathscr{L}_{\theta}$ was discovered; that is, for any $z=\left(z_{1}, z_{2}\right) \in \mathbb{R} \times \mathbb{R}^{n-1}$, one has

$$
z=\lambda_{1}(z) u_{z}^{1}+\lambda_{2}(z) u_{z}^{2}
$$

where

$$
\begin{gathered}
\lambda_{1}(z)=z_{1}-\left\|z_{2}\right\| \operatorname{ctan} \theta, \\
\lambda_{2}(z)=z_{1}+\left\|z_{2}\right\| \tan \theta, \\
u_{z}^{1}=\frac{1}{1+\operatorname{ctan}^{2} \theta}\left[\begin{array}{cc}
1 & 0 \\
0 & \operatorname{ctan} \theta \cdot I
\end{array}\right]\left[\begin{array}{c}
1 \\
-\bar{z}_{2}
\end{array}\right], \\
u_{z}^{2}=\frac{1}{1+\tan ^{2} \theta}\left[\begin{array}{cc}
1 & 0 \\
0 & \tan \theta \cdot I
\end{array}\right]\left[\begin{array}{c}
1 \\
\bar{z}_{2}
\end{array}\right],
\end{gathered}
$$

with $\bar{z}_{2}:=z_{2} /\left\|z_{2}\right\|$ if $z_{2} \neq 0$, and $\bar{z}_{2}$ being any vector $w \in \mathbb{R}^{n-1}$ satisfying $\|w\|=1$ if $z_{2}=0$. With this spectral decomposition (2), analogous to the so-called SOC-function $f^{\text {soc }}$ (see [35]) and SDP-function $f^{\text {mat }}$ (see $\left.[6,7]\right)$, we define a vectorvalued function associated with circular cone as below. More specifically, for $f: \mathbb{R} \rightarrow \mathbb{R}$, we define $f^{\mathscr{L}_{\theta}}: \mathbb{R}^{n} \rightarrow \mathbb{R}^{n}$ as

$$
f^{\mathscr{L}_{\theta}}(z)=f\left(\lambda_{1}(z)\right) u_{z}^{1}+f\left(\lambda_{2}(z)\right) u_{z}^{2} .
$$

It is not hard to see that $f^{\mathscr{L}_{\theta}}$ is well-defined for all $z$. In particular, if $z_{2}=0$, then

$$
f^{\mathscr{L}_{\theta}}(z)=\left[\begin{array}{c}
f\left(z_{1}\right) \\
0
\end{array}\right] .
$$


Note that when $\theta=45^{\circ}, \mathscr{L}_{\theta}$ reduces to the second-order cone (SOC) and the vector-valued function $f^{\mathscr{L}_{\theta}}$ defined as in (4) corresponds to the SOC-function $f^{\mathrm{soc}}$ given by

$$
\begin{array}{r}
f^{\mathrm{soc}}(x)=f\left(\lambda_{1}(x)\right) u_{x}^{(1)}+f\left(\lambda_{2}(x)\right) u_{x}^{(2)}, \\
\forall x=\left(x_{1}, x_{2}\right) \in \mathbb{R} \times \mathbb{R}^{n-1},
\end{array}
$$

where $\lambda_{i}(x)=x_{1}+(-1)^{i}\left\|x_{2}\right\|$ and $u_{x}^{(i)}=(1 / 2)\left(1,(-1)^{i} \bar{x}_{2}\right)^{T}$.

It is well known that the vector-valued function $f^{\mathrm{soc}}$ associated with second-order cone and matrix-valued function $f^{\text {mat }}$ associated with positive semidefinite cone play crucial role in the theory and numerical algorithm for secondorder cone programming and semidefinite programming, respectively. In particular, many properties of $f^{\text {soc }}$ and $f^{\text {mat }}$ are inherited from $f$, such as continuity, strictly continuity, directional differentiability, Fréchet differentiability, continuous differentiability, and semismoothness. It should be mentioned that, compared with second-order cone and positive semidefinite cone, $\mathscr{L}_{\theta}$ is a nonsymmetric cone. Hence a natural question arises whether these properties are still true for $f^{\mathscr{L}_{\theta}}$. In [1], the authors answer the questions from the following aspects:

(a) $f^{\mathscr{L}_{\theta}}$ is continuous at $z \in \mathbb{R}^{n}$ if and only if $f$ is continuous at $\lambda_{i}(z)$ for $i=1,2$;

(b) $f^{\mathscr{L}_{\theta}}$ is directionally differentiable at $z \in \mathbb{R}^{n}$ if and only if $f$ is directionally differentiable at $\lambda_{i}(z)$ for $i=1,2$;

(c) $f^{\mathscr{L}_{\theta}}$ is (Fréchet) differentiable at $z \in \mathbb{R}^{n}$ if and only if $f$ is (Fréchet) differentiable at $\lambda_{i}(z)$ for $i=1,2$;

(d) $f^{\mathscr{L}_{\theta}}$ is continuously differentiable at $z \in \mathbb{R}^{n}$ if and only if $f$ is continuously continuous at $\lambda_{i}(z)$ for $i=$ 1,2 ;

(e) $f^{\mathscr{L}_{\theta}}$ is strictly continuous at $z \in \mathbb{R}^{n}$ if and only if $f$ is strictly continuous at $\lambda_{i}(z)$ for $i=1,2$;

(f) $f^{\mathscr{L}_{\theta}}$ is Lipschitz continuous with constant $k>0$ if and only if $f$ is Lipschitz continuous with constant $k>0$;

(g) $f^{\mathscr{L}_{\theta}}$ is semismooth at $z$ if and only if $f$ is semismooth at $\lambda_{i}(z)$ for $i=1,2$.

In this paper, we further study some other properties associated with $f^{\mathscr{L}_{\theta}}$, such as Hölder continuity, $\rho$-order semismoothness, directionally differentiability in the Hadamard sense, the characterization of B-subdifferential, positive homogeneity, and boundedness. Of course, one may wonder whether $f^{\mathrm{soc}}$ and $f^{\mathscr{L}_{\theta}}$ always share the same properties. Indeed, they do not. There exists some property that holds for $f^{\text {soc }}$ and $f$ but fails for $f^{\mathscr{L}_{\theta}}$ and $f$. A counterexample is presented in the final section.

To end the third section, we briefly review our notations and some basic concepts which will be needed for subsequent analysis. First, we denote by $\mathbb{R}^{n}$ the space of $n$-dimensional real column vectors and let $e=(1,0, \ldots, 0) \in \mathbb{R}^{n}$. Given $x, y \in \mathbb{R}^{n}$, the Euclidean inner product and norm are $\langle x, y\rangle=$ $x^{T} y$ and $\|x\|=\sqrt{x^{T} x}$. For a linear mapping $H: \mathbb{R}^{n} \rightarrow \mathbb{R}^{m}$, its operator norm is $\|H\|:=\max _{\|x\|=1}\|H x\|$. For $\alpha \in \mathbb{R}$ and $s \in \mathbb{R}^{n}$, we write $s=O(\alpha)$ (resp., $s=o(\alpha)$ ) to means $\|s\| /|\alpha|$ is uniformly bounded (resp., tends to zero) as $\alpha \rightarrow 0$. In addition, given a function $F: \mathbb{R}^{n} \rightarrow \mathbb{R}^{m}$, we say the following:

(a) $F$ is Hölder continuous with exponent $\alpha \in(0,1]$, if

$$
[F]_{\alpha}:=\sup _{x \neq y} \frac{\|F(x)-F(y)\|}{\|x-y\|^{\alpha}}<+\infty
$$

(b) $F$ is directionally differentiable at $x \in \mathbb{R}^{n}$ in the Hadamard sense, if the directional derivative $F^{\prime}(x ; d)$ exists for all $d \in \mathbb{R}^{n}$ and

$$
F^{\prime}(x ; d)=\lim _{\substack{d^{\prime} \rightarrow d \\ t \downarrow 0}} \frac{F\left(x+t d^{\prime}\right)-F(x)}{t} ;
$$

(c) $F$ is $B$-differentiable (Bouligand-differentiable) at $x$, if $F$ is Lipschitz continuous near $x$ and directionally differentiable at $x$;

(d) if $F$ is strictly continuous (locally Lipschitz continuous), the generalized Jacobian $\partial F(x)$ is the convex hull of the $\partial_{B} F(x)$, where

$$
\partial_{B} F(x):=\left\{\lim _{z \rightarrow x} \nabla F(z) \mid z \in D_{F}\right\},
$$

where $D_{F}$ denotes the set of all differentiable points of F;

(e) $F$ is semismooth at $x$, if $F$ is strictly continuous near $x$, directionally differentiable at $x$, and for any $V \in$ $\partial F(x+h)$,

$$
F(x+h)-F(x)-V h=o(\|h\|) ;
$$

(f) $F$ is $\rho$-order semismooth at $x(\rho>0)$ if $F$ is semismooth at $x$ and for any $V \in \partial F(x+h)$,

$$
F(x+h)-F(x)-V h=O\left(\|h\|^{1+\rho}\right)
$$

in particular, we say $F$ is strongly semismooth if it is 1-order semismooth;

(g) $F$ is positively homogeneous with exponent $\alpha>0$, if

$$
F(k x)=k^{\alpha} F(x), \quad \forall x \in \mathbb{R}^{n}, k \geq 0 ;
$$

(h) $F$ is bounded if there exists a positive scalar $M>0$ such that

$$
\|F(x)\| \leq M, \quad \forall x \in \mathbb{R}^{n} .
$$




\section{Directional Differentiability, Strict Continuity, Hölder Continuity, and $B$-Differentiability}

This section is devoted to study the properties of directional differentiability, strict continuity, and Hölder continuity. The relationship of directional differentiability between $f^{\mathscr{L}_{\theta}}$ and $f$ has been given in [1, Theorem 3.2] without giving the exact formula of directional differentiability. Nonetheless, such formulas can be easily obtained from its proof. Here we just list them as follows.

Lemma 1. Let $f: \mathbb{R} \rightarrow \mathbb{R}$ and $f^{\mathscr{L}_{\theta}}$ be defined as in (4). Then, $f^{\mathscr{L}_{\theta}}$ is directionally differentiable at $z$ if and only if $f$ is directionally differentiable at $\lambda_{i}(z)$ for $i=1,2$. Moreover, for any $h=\left(h_{1}, h_{2}\right) \in \mathbb{R} \times \mathbb{R}^{n-1}$, we have

$$
\left(f^{\mathscr{L}_{\theta}}\right)^{\prime}(z ; h)=\left[\begin{array}{c}
f^{\prime}\left(z_{1} ; h_{1}\right) \\
0
\end{array}\right]=f^{\prime}\left(z_{1} ; h_{1}\right) e,
$$

when $z_{2}=0$ and $h_{2}=0$. Consider

$$
\begin{aligned}
\left(f^{\mathscr{L}_{\theta}}\right)^{\prime}(z ; h)= & \frac{1}{1+\operatorname{ctan}^{2} \theta} f^{\prime}\left(z_{1} ; h_{1}-\left\|h_{2}\right\| \operatorname{ctan} \theta\right) \\
& \times\left[\begin{array}{cc}
1 & 0 \\
0 & \operatorname{ctan} \theta \cdot I
\end{array}\right]\left[\begin{array}{c}
1 \\
-\frac{h_{2}}{\left\|h_{2}\right\|}
\end{array}\right] \\
& +\frac{1}{1+\tan ^{2} \theta} f^{\prime}\left(z_{1} ; h_{1}+\left\|h_{2}\right\| \tan \theta\right) \\
& \times\left[\begin{array}{cc}
1 & 0 \\
0 & \tan \theta \cdot I
\end{array}\right]\left[\begin{array}{c}
1 \\
\frac{h_{2}}{\left\|h_{2}\right\|}
\end{array}\right],
\end{aligned}
$$

when $z_{2}=0$ and $h_{2} \neq 0$; otherwise,

$$
\begin{aligned}
\left(f^{\mathscr{L}_{\theta}}\right)^{\prime}(z ; h)= & \frac{1}{1+\operatorname{ctan}^{2} \theta} f^{\prime}\left(\lambda_{1}(z) ; h_{1}-\frac{z_{2}^{T} h_{2}}{\left\|z_{2}\right\|} \operatorname{ctan} \theta\right) \\
& \times\left[\begin{array}{cc}
1 & 0 \\
0 & \operatorname{ctan} \theta \cdot I
\end{array}\right]\left[\begin{array}{c}
1 \\
-\frac{z_{2}}{\left\|z_{2}\right\|}
\end{array}\right] \\
& -\frac{\operatorname{ctan} \theta}{1+\operatorname{ctan}^{2} \theta} \frac{f\left(\lambda_{1}(z)\right)}{\left\|z_{2}\right\|} M_{z_{2}} h \\
& +\frac{1}{1+\tan ^{2} \theta} f^{\prime}\left(\lambda_{2}(z) ; h_{1}+\frac{z_{2}^{T} h_{2}}{\left\|z_{2}\right\|} \tan \theta\right) \\
& \times\left[\begin{array}{cc}
1 & 0 \\
0 & \tan \theta \cdot I
\end{array}\right]\left[\begin{array}{c}
1 \\
z_{2} \\
\left\|z_{2}\right\|
\end{array}\right] \\
& +\frac{\tan \theta}{1+\tan ^{2} \theta} \frac{f\left(\lambda_{2}(z)\right)}{\left\|z_{2}\right\|} M_{z_{2}} h,
\end{aligned}
$$

where

$$
M_{z_{2}}:=\left[\begin{array}{cc}
0 & 0 \\
0 & I-\frac{z_{2} z_{2}^{T}}{\left\|z_{2}\right\|^{2}}
\end{array}\right] .
$$

Lemma 2. Let $f: \mathbb{R} \rightarrow \mathbb{R}$ and $f^{\mathscr{L}_{\theta}}$ be defined as in (4). Then, the following hold.

(a) $f^{\mathscr{L}_{\theta}}$ is differentiable at $z$ if and only if $f$ is differentiable at $\lambda_{i}(z)$ for $i=1,2$. Moreover, if $z_{2}=0$, then

$$
\nabla f^{\mathscr{L}_{\theta}}(z)=f^{\prime}\left(z_{1}\right) I
$$

otherwise,

$$
\nabla f^{\mathscr{L}_{\theta}}(z)=\left[\begin{array}{cc}
\xi & \frac{\varrho z_{2}^{T}}{\left\|z_{2}\right\|} \\
\frac{\varrho z_{2}}{\left\|z_{2}\right\|} & a I+(\eta-a) \frac{z_{2} z_{2}^{T}}{\left\|z_{2}\right\|^{2}}
\end{array}\right],
$$

where

$$
\begin{gathered}
a=\frac{\tan \theta}{1+\tan ^{2} \theta} \frac{f\left(\lambda_{2}(z)\right)}{\left\|z_{2}\right\|}-\frac{\operatorname{ctan} \theta}{1+\operatorname{ctan}^{2} \theta} \frac{f\left(\lambda_{1}(z)\right)}{\left\|z_{2}\right\|} \\
=\frac{f\left(\lambda_{2}(z)\right)-f\left(\lambda_{1}(z)\right)}{\lambda_{2}(z)-\lambda_{1}(z)}, \\
\xi=\frac{f^{\prime}\left(\lambda_{1}(z)\right)}{1+\operatorname{ctan}^{2} \theta}+\frac{f^{\prime}\left(\lambda_{2}(z)\right)}{1+\tan ^{2} \theta}, \\
\eta=\xi-\varrho(\operatorname{ctan} \theta-\tan \theta), \\
\varrho=-\frac{\operatorname{ctan} \theta}{1+\operatorname{ctan}^{2} \theta} f^{\prime}\left(\lambda_{1}(z)\right)+\frac{\tan \theta}{1+\tan ^{2} \theta} f^{\prime}\left(\lambda_{2}(z)\right) .
\end{gathered}
$$

(b) $f^{\mathscr{L}_{\theta}}$ is continuously differentiable (smooth) at $z$ if and only if $f$ is continuously differentiable (smooth) at $\lambda_{i}(z)$ for $i=1,2$.

Note that the formula of gradient $\nabla f^{\mathscr{L}_{\theta}}$ given in $[1$, Theorem 3.3] and Lemma 2 is the same by using the following facts:

$$
\begin{gathered}
\frac{1}{1+\operatorname{ctan}^{2} \theta}=\sin ^{2} \theta, \quad \frac{1}{1+\tan ^{2} \theta}=\cos ^{2} \theta, \\
\frac{\operatorname{ctan} \theta}{1+\operatorname{ctan}^{2} \theta}=\frac{\tan \theta}{1+\tan ^{2} \theta}=\sin \theta \cos \theta .
\end{gathered}
$$

The following result indicating that $\lambda_{i}$ is Lipschitz continuous on $\mathbb{R}^{n}$ for $i=1,2$ will be used in proving the Lipschitz continuity between $f^{\mathscr{L}_{\theta}}$ and $f$.

Lemma 3. Let $z, y \in \mathbb{R}^{n}$ with spectral values $\lambda_{i}(z), \lambda_{i}(y)$, respectively. Then, we have

$$
\left|\lambda_{i}(z)-\lambda_{i}(y)\right| \leq \sqrt{2} \max \{\tan \theta, \operatorname{ctan} \theta\}\|z-y\|,
$$


Proof. First, we observe that

$$
\begin{aligned}
& \left|\lambda_{1}(z)-\lambda_{1}(y)\right| \\
& \quad=\left|z_{1}-\left\|z_{2}\right\| \operatorname{ctan} \theta-y_{1}+\left\|y_{2}\right\| \operatorname{ctan} \theta\right| \\
& \quad \leq\left|z_{1}-y_{2}\right|+\left\|z_{2}-y_{2}\right\| \operatorname{ctan} \theta \\
& \quad \leq \max \{1, \operatorname{ctan} \theta\}\left(\left|z_{1}-y_{1}\right|+\left\|z_{2}-y_{2}\right\|\right) \\
& \quad \leq \max \{1, \operatorname{ctan} \theta\} \sqrt{2} \sqrt{\left|z_{1}-y_{1}\right|^{2}+\left\|z_{2}-y_{2}\right\|^{2}} \\
& \quad=\max \{1, \operatorname{ctan} \theta\} \sqrt{2}\|z-y\| .
\end{aligned}
$$

Applying the similar argument to $\lambda_{2}$ yields

$$
\left|\lambda_{2}(z)-\lambda_{2}(y)\right| \leq \max \{1, \tan \theta\} \sqrt{2}\|z-y\| .
$$

Then, the desired result follows from the fact that $\max \{1$, $\operatorname{ctan} \theta, \tan \theta\} \max \{\operatorname{ctan} \theta, \tan \theta\}$.

Theorem 4. Let $f: \mathbb{R} \rightarrow \mathbb{R}$ and $f^{\mathscr{L}_{\theta}}$ be defined as in (4). Then, $f^{\mathscr{L}_{\theta}}$ is strictly continuous (local Lipschitz continuity) at $z$ if and only if $f$ is strictly continuous (local Lipschitz continuity) at $\lambda_{i}(z)$ for $i=1,2$.

Proof. " $\Leftarrow$ " Suppose that $f$ is strictly continuous at $\lambda_{i}(z)$, for $i=1,2$; that is, there exist $k_{i}>0$ and $\delta_{i}>0$, for $i=1,2$ such that

$$
\begin{array}{r}
|f(\tau)-f(\zeta)| \leq k_{i}|\tau-\zeta|, \\
\forall \tau, \zeta \in\left[\lambda_{i}(z)-\delta_{i}, \lambda_{i}(z)+\delta_{i}\right], i=1,2 .
\end{array}
$$

Let $\bar{\delta}:=\min \left\{\delta_{1}, \delta_{2}\right\}$ and $C:=\left[\lambda_{1}(z)-\bar{\delta}, \lambda_{1}(z)+\bar{\delta}\right] \cup\left[\lambda_{2}(z)-\right.$ $\left.\bar{\delta}, \lambda_{2}(z)+\bar{\delta}\right]$. Define

$$
\begin{aligned}
& \tilde{f}(\tau) \\
& :=\left\{\begin{array}{rr}
f(\tau) & \text { if } \tau \in C, \\
& \\
(1-t) f\left(\lambda_{1}(z)+\bar{\delta}\right) & \\
+t f\left(\lambda_{2}(z)-\bar{\delta}\right) & \text { if } \lambda_{1}(z)+\bar{\delta}<\lambda_{2} \\
& +t\left(\lambda_{2}(z)\right. \\
& \text { with } t \in(0,1) \\
f\left(\lambda_{1}(z)-\bar{\delta}\right) & \text { if } \tau<\lambda_{1}(z)-\bar{\delta} \\
f\left(\lambda_{2}(z)+\bar{\delta}\right) & \text { if } \tau>\lambda_{2}(z)+\bar{\delta} .
\end{array}\right.
\end{aligned}
$$

Clearly, $\tilde{f}$ is Lipschitz continuous on $\mathbb{R}$; that is, there exists $k>0$ such that $\operatorname{lip} \widetilde{f}(\tau) \leq k$, for all $\tau \in \mathbb{R}$. Since $\widetilde{C}:=\operatorname{conv}(C)$ is compact, according to [6, Lemma 4.5] or [5, Lemma 3], there exist continuously differentiable functions $f^{v}: \mathbb{R} \rightarrow \mathbb{R}$ for $v=1,2, \ldots$ converging uniformly to $\widetilde{f}$ on $\widetilde{C}$ such that

$$
\left|\left(f^{v}\right)^{\prime}(\tau)\right| \leq k \quad \forall \tau \in \widetilde{C}, \forall v
$$

Now, let $:=\bar{\delta} /(\sqrt{2} \max \{\tan \theta, \operatorname{ctan} \theta\})$. Then, from Lemma 3, we know that $\widetilde{C}$ contains all spectral values of $y \in \mathbb{B}(z, \delta)$. Therefore, for any $w \in \mathbb{B}(z, \delta)$, we have $\lambda_{i}(w) \in \widetilde{C}$ for $i=1,2$ and

$$
\begin{aligned}
\left\|\left(f^{v}\right)^{\mathscr{L}_{\theta}}(w)-f^{\mathscr{L}_{\theta}}(w)\right\|^{2} \\
=\|\left[f^{v}\left(\lambda_{1}(w)\right)-f\left(\lambda_{1}(w)\right)\right] u_{w}^{1} \\
\quad+\left[f^{v}\left(\lambda_{2}(w)\right)-f\left(\lambda_{2}(w)\right)\right] u_{w}^{2} \|^{2} \\
=\left[f^{v}\left(\lambda_{1}(w)\right)-f\left(\lambda_{1}(w)\right)\right]^{2}\left\|u_{w}^{1}\right\|^{2} \\
\quad+\left[f^{v}\left(\lambda_{2}(w)\right)-f\left(\lambda_{2}(w)\right)\right]^{2}\left\|u_{w}^{2}\right\|^{2} \\
=\frac{1}{1+\operatorname{ctan}^{2} \theta}\left|f^{v}\left(\lambda_{1}(w)\right)-f\left(\lambda_{1}(w)\right)\right|^{2} \\
\quad+\frac{1}{1+\tan ^{2} \theta}\left|f^{v}\left(\lambda_{2}(w)\right)-f\left(\lambda_{2}(w)\right)\right|^{2},
\end{aligned}
$$

where we have used the facts that $\left\|u_{w}^{1}\right\|=1 / \sqrt{1+\operatorname{ctan}^{2} \theta}$, $\left\|u_{w}^{2}\right\|=1 / \sqrt{1+\tan ^{2} \theta}$, and $\left\langle u_{w}^{1}, u_{w}^{2}\right\rangle=0$. Since $\left\{f^{v}\right\}_{v=1}^{\infty}$ converges uniformly to $f$ on $\widetilde{C}$, the above equations show that $\left\{\left(f^{v}\right)^{\mathscr{L}_{\theta}}\right\}_{v=1}^{\infty}$ converges uniformly to $f^{\mathscr{L}_{\theta}}$ on $\mathbb{B}(z, \delta)$. If $w_{2}=0$, then it follows from Lemma 2 that $\nabla\left(f^{v}\right)^{\mathscr{L}_{\theta}}(w)=\left(f^{v}\right)^{\prime}\left(w_{1}\right) I$. Hence it follows from (27) that

$$
\left\|\nabla\left(f^{v}\right)^{\mathscr{L}_{\theta}}(w)\right\|=\left|\left(f^{\nu}\right)^{\prime}\left(w_{1}\right)\right| \leq k
$$

since in this case $\lambda_{i}(w)=w_{1} \in \widetilde{C}$. If $w_{2} \neq 0$, then

$$
\begin{aligned}
& \nabla\left(f^{v}\right)^{\mathscr{L}_{\theta}}(w) \\
& =\left[\begin{array}{cc}
\xi & \frac{\varrho w_{2}^{T}}{\left\|w_{2}\right\|} \\
\frac{\varrho w_{2}}{\left\|w_{2}\right\|} & a I+(\xi-\varrho(\operatorname{ctan} \theta-\tan \theta)-a) \frac{w_{2} w_{2}^{T}}{\left\|w_{2}\right\|^{2}}
\end{array}\right] \\
& =\left[\begin{array}{cc}
\xi & \frac{\varrho w_{2}^{T}}{\left\|w_{2}\right\|} \\
\frac{\varrho w_{2}}{\left\|w_{2}\right\|} & a I+(\xi-a) \frac{w_{2} w_{2}^{T}}{\left\|w_{2}\right\|^{2}}
\end{array}\right] \\
& +\left[\begin{array}{cc}
0 & 0 \\
0 & {[-\varrho(\operatorname{ctan} \theta-\tan \theta)] \frac{w_{2} w_{2}^{T}}{\left\|w_{2}\right\|^{2}}}
\end{array}\right] \\
& =\left[\begin{array}{cc}
\xi & \frac{\varrho w_{2}^{T}}{\left\|w_{2}\right\|} \\
\frac{\varrho w_{2}}{\left\|w_{2}\right\|} & \xi I
\end{array}\right]+(a-\xi)\left[\begin{array}{cc}
0 & 0 \\
0 & I-\frac{w_{2} w_{2}^{T}}{\left\|w_{2}\right\|^{2}}
\end{array}\right] \\
& -\varrho(\operatorname{ctan} \theta-\tan \theta)\left[\begin{array}{cc}
0 & 0 \\
0 & \frac{w_{2} w_{2}^{T}}{\left\|w_{2}\right\|^{2}}
\end{array}\right],
\end{aligned}
$$


where $a, \xi, \varrho$ are given as in (20) with $\lambda_{i}(z)$ replaced by $\lambda_{i}(w)$ for $i=1,2$ and $f$ replaced by $f^{v}$. For simplicity of notations, let us denote

$$
\begin{gathered}
A:=\left[\begin{array}{cc}
\xi & \frac{\varrho w_{2}^{T}}{\left\|w_{2}\right\|} \\
\frac{\varrho w_{2}}{\left\|w_{2}\right\|} & \xi I
\end{array}\right]+(a-\xi)\left[\begin{array}{cc}
0 & 0 \\
0 & I-\frac{w_{2} w_{2}^{T}}{\left\|w_{2}\right\|^{2}}
\end{array}\right], \\
B:=-\varrho(\operatorname{ctan} \theta-\tan \theta)\left[\begin{array}{cc}
0 & 0 \\
0 & \frac{w_{2} w_{2}^{T}}{\left\|w_{2}\right\|^{2}}
\end{array}\right] .
\end{gathered}
$$

Note that

$$
|a|=\left|\frac{f^{v}\left(\lambda_{2}(w)\right)-f^{v}\left(\lambda_{1}(w)\right)}{\lambda_{2}(w)-\lambda_{1}(w)}\right| \leq k,
$$

where the inequality comes from the fact that $f^{v}$ is continuously differentiable on $\widetilde{C}$ and (27). Besides, we also note that

$$
\begin{aligned}
|\xi| & =\left|\frac{\left(f^{v}\right)^{\prime}\left(\lambda_{1}(w)\right)}{1+\operatorname{ctan}^{2} \theta}+\frac{\left(f^{v}\right)^{\prime}\left(\lambda_{2}(w)\right)}{1+\tan ^{2} \theta}\right| \\
& \leq \frac{1}{1+\operatorname{ctan}^{2} \theta}\left|\left(f^{v}\right)^{\prime}\left(\lambda_{1}(w)\right)\right|+\frac{1}{1+\tan ^{2} \theta}\left|\left(f^{v}\right)^{\prime}\left(\lambda_{2}(w)\right)\right| \\
& \leq\left[\frac{1}{1+\operatorname{ctan}^{2} \theta}+\frac{1}{1+\tan ^{2} \theta}\right] k=k,
\end{aligned}
$$

$$
\begin{aligned}
|\varrho| & =\left|-\frac{\operatorname{ctan} \theta}{1+\operatorname{ctan}^{2} \theta}\left(f^{v}\right)^{\prime}\left(\lambda_{1}(w)\right)+\frac{\tan \theta}{1+\tan ^{2} \theta}\left(f^{v}\right)^{\prime}\left(\lambda_{2}(w)\right)\right| \\
& \leq\left[\left|-\frac{\operatorname{ctan} \theta}{1+\operatorname{ctan}^{2} \theta}\right|+\left|\frac{\tan \theta}{1+\tan ^{2} \theta}\right|\right] k \\
& =\left[\frac{\operatorname{ctan} \theta}{1+\operatorname{ctan}^{2} \theta}+\frac{\tan \theta}{1+\tan ^{2} \theta}\right] k \\
& =\frac{2 \tan \theta}{1+\tan ^{2} \theta} k \leq k .
\end{aligned}
$$

(i) For $\varrho=0$, then $\nabla\left(f^{v}\right)^{\mathscr{L}_{\theta}}(w)$ takes the form of $\xi I+(a-$ $\xi) M_{w_{2}}$ whose eigenvalues are $\xi$ and $a$ by [5, Lemma 1]. In other words, in this case, we get from (32) and (33) that

$$
\left\|\nabla\left(f^{v}\right)^{\mathscr{L}_{\theta}}(w)\right\|=\max \{|a|,|\xi|\} \leq k .
$$

(ii) For $\varrho \neq 0$, since $B=-\varrho(\operatorname{ctan} \theta-\tan \theta)\left(0, w_{2} /\left\|w_{2}\right\|\right)^{T}$ $\left(0, w_{2} /\left\|w_{2}\right\|\right)$, the eigenvalues of $B$ are $-\varrho(\operatorname{ctan} \theta-$ $\tan \theta)$ and 0 with multiplicity $n-1$. Note that

$$
|\varrho(\operatorname{ctan} \theta-\tan \theta)|
$$

$$
=\left|\frac{1-\operatorname{ctan}^{2} \theta}{1+\operatorname{ctan}^{2} \theta}\left(f^{v}\right)^{\prime}\left(\lambda_{1}(w)\right)+\frac{1-\tan ^{2} \theta}{1+\tan ^{2} \theta}\left(f^{v}\right)^{\prime}\left(\lambda_{2}(w)\right)\right|
$$

$$
\begin{aligned}
& \leq\left[\left|\frac{1-\operatorname{ctan}^{2} \theta}{1+\operatorname{ctan}^{2} \theta}\right|+\left|\frac{1-\tan ^{2} \theta}{1+\tan ^{2} \theta}\right|\right] k \\
& =\left|\frac{\operatorname{ctan}^{2} \theta-1}{1+\operatorname{ctan}^{2} \theta}+\frac{1-\tan ^{2} \theta}{1+\tan ^{2} \theta}\right| k \\
& =2\left|\frac{1-\tan ^{2} \theta}{1+\tan ^{2} \theta}\right| k \leq 2 k .
\end{aligned}
$$

Note that

$$
\begin{aligned}
A & =\frac{\varrho}{\left\|w_{2}\right\|} L_{\widetilde{w}}+(a-\xi) M_{w_{2}} \\
& =\frac{\varrho}{\left\|w_{2}\right\|}\left[L_{\widetilde{w}}+(a-\xi) \frac{\left\|w_{2}\right\|}{\varrho} M_{\widetilde{w}_{2}}\right],
\end{aligned}
$$

where $\widetilde{w}=\left(\xi\left\|w_{2}\right\| / \varrho, w_{2}\right)$ and

$$
L_{\widetilde{w}}:=\left[\begin{array}{cc}
\widetilde{w}_{1} & \widetilde{w}_{2}^{T} \\
\widetilde{w}_{2} & \widetilde{w}_{1} I
\end{array}\right] .
$$

In this case the matrix $A$ has eigenvalues of $\xi \pm \varrho$ and $a$ with multiplicity $n-2$. Hence,

$$
\begin{aligned}
& \left\|\nabla\left(f^{v}\right)^{\mathscr{L}_{\theta}}(w)\right\| \\
& \quad \leq \max \{|\xi+\varrho|,|\xi-\varrho|,|a|\}+|\varrho(\operatorname{ctan} \theta-\tan \theta)| \\
& \quad \leq \max \{|\xi|+|\varrho|,|a|\}+|\varrho(\operatorname{ctan} \theta-\tan \theta)| \leq 4 k,
\end{aligned}
$$

where the last step is due to (32), (33), (34), and (36).

Putting (29), (35), and (39) together, we know that

$$
\left\|\nabla\left(f^{v}\right)^{\mathscr{L}_{\theta}}(w)\right\| \leq 4 k \quad \forall w \in \mathbb{B}(z, \delta), \forall v .
$$

Fix any $x, y \in \mathbb{B}(z, \delta)$ with $x \neq y$. Since $\left\{\left(f^{v}\right)^{\mathscr{L}_{\theta}}\right\}_{v=1}^{\infty}$ converges uniformly to $f^{\mathscr{L}_{\theta}}$ on $\mathbb{B}(z, \delta)$, then for any $\epsilon>0$ there exists $v_{0}$ such that

$$
\left\|\left(f^{v}\right)^{\mathscr{L}_{\theta}}(w)-f^{\mathscr{L}_{\theta}}(w)\right\| \leq \epsilon, \quad \forall w \in \mathbb{B}(z, \delta), \forall v \geq v_{0} .
$$

Since $f^{v}$ is continuously differentiable, $\left(f^{v}\right)^{\mathscr{L}_{\theta}}$ is continuously differentiable by Lemma 2 . Thus,

$$
\begin{aligned}
\left\|f^{\mathscr{L}_{\theta}}(x)-f^{\mathscr{L}_{\theta}}(y)\right\| \\
=\| f^{\mathscr{L}_{\theta}}(x)-\left(f^{v}\right)^{\mathscr{L}_{\theta}}(x)+\left(f^{v}\right)^{\mathscr{L}_{\theta}}(x)-\left(f^{v}\right)^{\mathscr{L}_{\theta}}(y) \\
\quad+\left(f^{v}\right)^{\mathscr{L}_{\theta}}(y)-f^{\mathscr{L}_{\theta}}(y) \| \\
\leq\left\|f^{\mathscr{L}_{\theta}}(x)-\left(f^{v}\right)^{\mathscr{L}_{\theta}}(x)\right\|+\left\|\left(f^{v}\right)^{\mathscr{L}_{\theta}}(x)-\left(f^{v}\right)^{\mathscr{L}_{\theta}}(y)\right\| \\
\quad+\left\|\left(f^{v}\right)^{\mathscr{L}_{\theta}}(y)-f^{\mathscr{L}_{\theta}}(y)\right\| \\
\leq 2 \epsilon+\left\|\int_{0}^{1} \nabla\left(f^{v}\right)^{\mathscr{L}_{\theta}}(y+t(x-y))(x-y) d t\right\| \\
\leq 2 \epsilon+4 k\|x-y\| .
\end{aligned}
$$


Because $\epsilon>0$ is arbitrary, this ensures that

$$
\left\|f^{\mathscr{L}_{\theta}}(x)-f^{\mathscr{L}_{\theta}}(y)\right\| \leq 4 k\|x-y\| \quad \forall x, y \in \mathbb{B}(z, \delta),
$$

which says $f^{\mathscr{L}_{\theta}}$ is strictly continuous at $z$.

“ $\Rightarrow$ " Suppose that $f^{\mathscr{L}_{\theta}}$ is strictly continuous at $z$, then there exist $k>0$ and $\delta>0$ such that

$$
\left\|f^{\mathscr{L}_{\theta}}(x)-f^{\mathscr{L}_{\theta}}(y)\right\| \leq k\|x-y\| \quad \forall x, y \in \mathbb{B}(z, \delta) .
$$

Case 1. $z_{2} \neq 0$. Take $\theta, \mu \in\left[\lambda_{1}(z)-\delta_{1}, \lambda_{1}(z)+\delta_{1}\right]$ with $\delta_{1}:=$ $\min \left\{\delta, \lambda_{2}(z)-\lambda_{1}(z)\right\}$. Let

$$
x:=\theta u_{z}^{1}+\lambda_{2}(z) u_{z}^{2}, \quad y:=\mu u_{z}^{1}+\lambda_{2}(z) u_{z}^{2} .
$$

Then, $\|x-z\| \leq \delta$ and $\|y-z\| \leq \delta$ and it follows from (44) that

$$
\begin{aligned}
|f(\theta)-f(\mu)| & =\frac{1}{\left\|u_{z}^{1}\right\|}\left\|f^{\mathscr{L}_{\theta}}(x)-f^{\mathscr{L}_{\theta}}(y)\right\| \leq \frac{k}{\left\|u_{z}^{1}\right\|}\|x-y\| \\
& =\frac{k}{\left\|u_{z}^{1}\right\|}|\theta-\mu|\left\|u_{z}^{1}\right\|=k|\theta-\mu|,
\end{aligned}
$$

which says $f$ is strictly continuous at $\lambda_{1}(z)$. The similar argument shows the strict continuity of $f$ at $\lambda_{2}(z)$.

Case 2. $z_{2}=0$. For any $\theta, \mu \in\left[z_{1}-\delta, z_{1}+\delta\right]$, we have $\|\theta e-z\|=$ $\left|\theta-z_{1}\right| \leq \delta$ and $\|\mu e-z\| \leq \delta$ as well; that is, $\theta e$, $\mu e \in \mathbb{B}(z, \delta)$. It then follows from (44) that

$$
\begin{aligned}
|f(\theta)-f(\mu)| & =\left\|\left(\begin{array}{c}
f(\theta)-f(\mu) \\
0
\end{array}\right)\right\|=\left\|f^{\mathscr{L}_{\theta}}(\theta e)-f^{\mathscr{L}_{\theta}}(\mu e)\right\| \\
& \leq k\|\theta e-\mu e\|=k|\theta-\mu| .
\end{aligned}
$$

This means $f$ is strictly continuous at $\lambda_{i}(z)=z_{1}$ for $i=1,2$.

Remark 5. As mentioned in Section 1, the strict continuity between $f^{\mathscr{L}_{\theta}}$ and $f$ has been given in [1, Theorem 3.5]. Here we provide an alternative proof, since our analysis technique is different from that in [1, Theorem 3.5]. In particular, we achieve an estimate regarding $\left\|\nabla\left(f^{v}\right)^{\mathscr{L}_{\theta}}\right\|$ via its eigenvalues, which may have other applications.

According to Lemma 1 and Theorem 4, we obtain the following result immediately.

Theorem 6. Let $f: \mathbb{R} \rightarrow \mathbb{R}$ and $f^{\mathscr{L}_{\theta}}$ be defined as in (4). Then, $f^{\mathscr{L}_{\theta}}$ is B-differentiable at $z$ if and only if $f$ is B-differentiable at $\lambda_{i}(z)$, for $i=1,2$.

Next, inspired by $[8,9]$, we further study the Hölder continuity relation between $f$ and $f^{\mathscr{L}_{\theta}}$.
Theorem 7. Let $f: \mathbb{R} \rightarrow \mathbb{R}$ and $f^{\mathscr{L}_{\theta}}$ be defined as in (4). Then, $f^{\mathscr{L}_{\theta}}$ is Hölder continuous with exponent $\alpha \in(0,1]$ if and only if $f$ is Hölder continuous with exponent $\alpha \in(0,1]$.

Proof. " $\Leftarrow$ " Suppose that $f$ is Hölder continuous with exponent $\alpha \in(0,1]$. To proceed the proof, we consider the following two cases.

Case $1 . z_{2} \neq 0$ and $y_{2} \neq 0$. We assume without loss of generality that $\left\|z_{2}\right\| \geq\left\|y_{2}\right\|$. Thus,

$$
\begin{aligned}
& \left\|f^{\mathscr{L}_{\theta}}(z)-f^{\mathscr{L}_{\theta}}(y)\right\| \\
& =\| f\left(\lambda_{1}(z)\right) u_{z}^{1}+f\left(\lambda_{2}(z)\right) u_{z}^{2}-f\left(\lambda_{1}(y)\right) u_{y}^{1} \\
& \quad-f\left(\lambda_{2}(y)\right) u_{y}^{2} \| \\
& =\| f\left(\lambda_{1}(z)\right)\left[u_{z}^{1}-u_{y}^{1}\right]+f\left(\lambda_{2}(z)\right)\left[u_{z}^{2}-u_{y}^{2}\right] \\
& +\left[f\left(\lambda_{1}(z)\right)-f\left(\lambda_{1}(y)\right)\right] u_{y}^{1} \\
& +\left[f\left(\lambda_{2}(z)\right)-f\left(\lambda_{2}(y)\right)\right] u_{y}^{2} \| \\
& \leq\left\|f\left(\lambda_{1}(z)\right)\left[u_{z}^{1}-u_{y}^{1}\right]+f\left(\lambda_{2}(z)\right)\left[u_{z}^{2}-u_{y}^{2}\right]\right\| \\
& +\left|f\left(\lambda_{1}(z)\right)-f\left(\lambda_{1}(y)\right)\right| \cdot\left\|u_{y}^{1}\right\| \\
& +\left|f\left(\lambda_{2}(z)\right)-f\left(\lambda_{2}(y)\right)\right| \cdot\left\|u_{y}^{2}\right\| \cdot .
\end{aligned}
$$

Let us analyze each term in the above inequality. First, we look into the first term:

$$
\begin{aligned}
\| f & \left(\lambda_{1}(z)\right)\left[u_{z}^{1}-u_{y}^{1}\right]+f\left(\lambda_{2}(z)\right)\left[u_{z}^{2}-u_{y}^{2}\right] \| \\
& =\frac{\tan \theta}{1+\tan ^{2} \theta}\left|f\left(\lambda_{1}(z)\right)-f\left(\lambda_{2}(z)\right)\right| \cdot \| \frac{z_{2}}{\left\|z_{2}\right\|}-\frac{y_{2}}{\left\|y_{2}\right\| \|} \\
& \leq \frac{\tan \theta}{1+\tan ^{2} \theta}[f]_{\alpha}\left|\lambda_{1}(z)-\lambda_{2}(z)\right|^{\alpha} \| \frac{z_{2}}{\left\|z_{2}\right\|}-\frac{y_{2}}{\left\|y_{2}\right\| \|} \\
& =\frac{\tan \theta}{1+\tan ^{2} \theta}[f]_{\alpha}(\tan \theta+\operatorname{ctan} \theta)^{\alpha}\left\|z_{2}\right\|^{\alpha} \cdot\left\|\frac{z_{2}}{\left\|z_{2}\right\|}-\frac{y_{2}}{\left\|y_{2}\right\| \|}\right\| \\
& \leq \frac{\tan \theta}{1+\tan ^{2} \theta}[f]_{\alpha}(\tan \theta+\operatorname{ctan} \theta)^{\alpha}\left\|z_{2}\right\|^{\alpha} \frac{2}{\left\|z_{2}\right\|}\left\|z_{2}-y_{2}\right\| \\
& =2 \frac{\tan ^{2} \theta}{1+\tan ^{2} \theta}[f]_{\alpha}(\tan \theta+\operatorname{ctan} \theta)^{\alpha}\left\|\frac{z_{2}-y_{2}}{\left\|z_{2}\right\|}\right\|^{1-\alpha}\left\|z_{2}-y_{2}\right\|^{\alpha} \\
& \leq \frac{\tan ^{\alpha}}{1+\tan ^{2} \theta}[f]_{\alpha}(\tan \theta+\operatorname{ctan} \theta)^{\alpha} 2^{2-\alpha}\left\|z_{2}-y_{2}\right\|^{\alpha} \\
& \leq \frac{\tan ^{2} \theta}{1+\tan ^{2} \theta}[f]_{\alpha}(\tan \theta+\operatorname{ctan} \theta)^{\alpha} 2^{2-\alpha}\|z-y\|^{\alpha},
\end{aligned}
$$

where the first inequality is due to the Hölder continuity of $f$, the second inequality comes from the fact that $\left\|\left(z_{2} /\left\|z_{2}\right\|\right)-\left(y_{2} /\left\|y_{2}\right\|\right)\right\| \leq\left(2 /\left\|z_{2}\right\|\right)\left\|z_{2}-y_{2}\right\|$ (cf. [8, Lemma $2.2]$ ), and the third inequality follows from the fact that 
$\left\|z_{2}-y_{2}\right\| \leq\left\|z_{2}\right\|+\left\|y_{2}\right\| \leq 2\left\|z_{2}\right\|$ (since $\left.\left\|y_{2}\right\| \leq\left\|z_{2}\right\|\right)$. Next, we look into the second term:

$$
\begin{aligned}
& \left|f\left(\lambda_{1}(z)\right)-f\left(\lambda_{1}(y)\right)\right|\left\|u_{y}^{1}\right\| \\
& \quad \leq[f]_{\alpha}\left|\lambda_{1}(z)-\lambda_{1}(y)\right|^{\alpha} \frac{1}{\sqrt{1+\operatorname{ctan}^{2} \theta}} \\
& \quad \leq[f]_{\alpha}(\sqrt{2} \max \{\tan \theta, \operatorname{ctan} \theta\})^{\alpha}\|z-y\|^{\alpha} .
\end{aligned}
$$

Similarly, the third term also satisfies

$$
\begin{aligned}
& \left|f\left(\lambda_{2}(z)\right)-f\left(\lambda_{2}(y)\right)\right|\left\|u_{y}^{2}\right\| \\
& \quad \leq[f]_{\alpha}\left|\lambda_{2}(z)-\lambda_{2}(y)\right|^{\alpha} \frac{1}{\sqrt{1+\tan ^{2} \theta}} \\
& \quad \leq[f]_{\alpha}(\sqrt{2} \max \{\tan \theta, \operatorname{ctan} \theta\})^{\alpha}\|z-y\|^{\alpha} .
\end{aligned}
$$

Combining (49)-(51) proves that $f^{\mathscr{L}_{\theta}}$ is Hölder continuous with exponent $\alpha \in(0,1]$.

Case 2. Either $z_{2}=0$ or $y_{2}=0$. In this case, we take $u_{z}^{i}=u_{y}^{i}$, for $i=1,2$ according to the spectral decomposition. Therefore, we obtain

$$
\begin{aligned}
& \left\|f^{\mathscr{L}_{\theta}}(z)-f^{\mathscr{L}_{\theta}}(y)\right\| \\
& =\| f\left(\lambda_{1}(z)\right) u_{z}^{1}+f\left(\lambda_{2}(z)\right) u_{z}^{2}-f\left(\lambda_{1}(y)\right) u_{y}^{1} \\
& \quad-f\left(\lambda_{2}(y)\right) u_{y}^{2} \| \\
& =\|\left[f\left(\lambda_{1}(z)\right)-f\left(\lambda_{1}(y)\right)\right] u_{z}^{1} \\
& \quad+\left[f\left(\lambda_{2}(z)\right)-f\left(\lambda_{2}(y)\right)\right] u_{z}^{2} \| \\
& \leq\left|f\left(\lambda_{1}(z)\right)-f\left(\lambda_{1}(y)\right)\right| \cdot\left\|u_{z}^{1}\right\| \\
& \quad+\left|f\left(\lambda_{2}(z)\right)-f\left(\lambda_{2}(y)\right)\right| \cdot\left\|u_{z}^{2}\right\| \\
& \leq \quad[f]_{\alpha}\left|\lambda_{1}(z)-\lambda_{1}(y)\right|^{\alpha} \frac{1}{\sqrt{1+\operatorname{ctan}^{2} \theta}} \\
& \quad+[f]_{\alpha}\left|\lambda_{2}(y)-\lambda_{2}(z)\right|^{\alpha} \frac{1}{\sqrt{1+\tan ^{2} \theta}} \\
& \leq 2[f]_{\alpha}(\sqrt{2} \max \{\tan \theta, \operatorname{ctan} \theta\})^{\alpha}\|z-y\|^{\alpha}
\end{aligned}
$$

which says $f^{\mathscr{L}_{\theta}}$ is Hölder continuous.

“ $\Rightarrow$ " Recall that $f^{\mathscr{L}_{\theta}}(\tau e)=(f(\tau), 0)^{T}$. Hence, for any $\tau, \zeta \in \mathbb{R}$

$$
\begin{aligned}
|f(\tau)-f(\zeta)| & =\left\|f^{\mathscr{L}_{\theta}}(\tau e)-f^{\mathscr{L}_{\theta}}(\zeta e)\right\| \\
& \leq\left[f^{\mathscr{L}_{\theta}}\right]_{\alpha} \cdot\|\tau e-\zeta e\|^{\alpha}=\left[f^{\mathscr{L}_{\theta}}\right]_{\alpha} \cdot|\tau-\zeta|^{\alpha}
\end{aligned}
$$

which says $f$ is Hölder continuous.

\section{3. $\rho$-Order Semismoothness and $B$-Subdifferential Formula}

The property of semismoothness plays an important role in nonsmooth Newton methods $[10,11]$. For more information on semismooth functions, see [12-15]. The relationship of semismooth between $f^{\mathscr{L}_{\theta}}$ and $f$ has been given in [1, Theorem 4.1]. But the exact formula of the $B$-subdifferential $\partial_{B}\left(f^{\mathscr{L}_{\theta}}\right)$ is not presented. Hence the main aim of this section is twofold: one is establishing the exact formula of $B$ subdifferential; another is studing the $\rho$-order semismoothness for $\rho>0$.

Lemma 8. Define $\psi(z)=\|z\|$ and $\Phi(z)=z /\|z\|$ for $z \neq 0$. Then, $\psi$ and $\Phi$ are strongly semismooth at $z \neq 0$.

Proof. Since $z \neq 0$, it is clear that $\psi$ and $\Phi$ are twice continuously differentiable and hence the gradient is Lipschitz continuous near $z$. Therefore, $\psi$ and $\Phi$ are strongly semismooth at $z$, see [16, Proposition 7.4.5].

The relationship of $\rho$-order semismoothness between $f^{\mathscr{L}_{\theta}}$ and $f$ is given below. Recall from [7] that in the definition of $\rho$-order semismooth, we can restrict $x+h$ in (11) belonging to differentiable points.

Theorem 9. Let $f: \mathbb{R} \rightarrow \mathbb{R}$ and $f^{\mathscr{L}_{\theta}}$ be defined as in (4). Given $\rho>0$, then the following statements hold.

(a) If $f$ is $\rho$-order semismooth at $\lambda_{i}(z)$ for $i=1,2$, then $f^{\mathscr{L}_{\theta}}$ is $\min \{1, \rho\}$-order semismooth at $z$.

(b) If $f^{\mathscr{L}_{\theta}}$ is $\rho$-order semismooth at $z$, then $f$ is $\rho$-semismooth at $\lambda_{i}(z)$ for $i=1,2$.

(c) For $z_{2}=0, f^{\mathscr{L}_{\theta}}$ is $\rho$-semismooth at $z$ if and only if $f$ is $\rho$-order semismooth at $\lambda_{i}(z)=z_{1}$ for $i=1,2$.

Proof. (a) Take $h \in \mathbb{R}^{n}$ satisfying $z+h \in D_{f^{\mathscr{L}_{\theta}}}$. We consider the following two cases to complete the proof.

Case 1. For $z_{2} \neq 0, z_{2}+h_{2} \neq 0$ as $h$ is sufficiently close to 0 . Since $z+h \in D_{f^{\mathscr{L}_{\theta}}}$, we know that $\lambda_{i}(z+h) \in D_{f}$ for $i=1,2$ by Lemma 2. Then, according to Lemma 1, the first component of

$$
f^{\mathscr{L}_{\theta}}(z+h)-f^{\mathscr{L}_{\theta}}(z)-\left(f^{\mathscr{L}_{\theta}}\right)^{\prime}(z+h ; h)
$$

is expressed as

$$
\begin{aligned}
& \frac{f\left(\lambda_{1}(z+h)\right)}{1+\operatorname{ctan}^{2} \theta}-\frac{f\left(\lambda_{1}(z)\right)}{1+\operatorname{ctan}^{2} \theta}-\frac{1}{1+\operatorname{ctan}^{2} \theta} \\
& \times f^{\prime}\left(\lambda_{1}(z+h) ; h_{1}-\frac{\left(z_{2}+h_{2}\right)^{T} h_{2}}{\left\|z_{2}+h_{2}\right\|} \operatorname{ctan} \theta\right) \\
& \quad+\frac{f\left(\lambda_{2}(z+h)\right)}{1+\tan ^{2} \theta}-\frac{f\left(\lambda_{2}(z)\right)}{1+\tan ^{2} \theta}-\frac{1}{1+\tan ^{2} \theta} \\
& \quad \times f^{\prime}\left(\lambda_{2}(z+h) ; h_{1}+\frac{\left(z_{2}+h_{2}\right)^{T} h_{2}}{\left\|z_{2}+h_{2}\right\|} \tan \theta\right) .
\end{aligned}
$$


Because $\|\cdot\|$ is continuously differentiable over $z_{2} \neq 0$, it is strongly semismooth at $z_{2}$ by Lemma 8 . Therefore,

$$
\begin{aligned}
\left\|z_{2}+h_{2}\right\| & =\left\|z_{2}\right\|+\frac{\left(z_{2}+h_{2}\right)^{T} h_{2}}{\left\|z_{2}+h_{2}\right\|}+O\left(\left\|h_{2}\right\|^{2}\right) \\
& =\left\|z_{2}\right\|+\frac{\left(z_{2}+h_{2}\right)^{T} h_{2}}{\left\|z_{2}+h_{2}\right\|}+O\left(\|h\|^{2}\right) .
\end{aligned}
$$

Combining this and the $\rho$-semismoothness of $f$ at $\lambda_{1}(z)$, we have

$$
\begin{aligned}
f\left(\lambda_{1}(z+h)\right) & f\left(\lambda_{1}(z)\right)+f^{\prime}\left(\lambda_{1}(z+h)\right)\left(\lambda_{1}(z+h)-\lambda_{1}(z)\right) \\
& +O\left(\left|\lambda_{1}(z+h)-\lambda_{1}(z)\right|^{1+\rho}\right) \\
= & f\left(\lambda_{1}(z)\right)+f^{\prime}\left(\lambda_{1}(z+h)\right)\left(\lambda_{1}(z+h)-\lambda_{1}(z)\right) \\
& +O\left(\|h\|^{1+\rho}\right) \\
= & f\left(\lambda_{1}(z)\right)+f^{\prime}\left(\lambda_{1}(z+h)\right) \\
& \times\left(h_{1}-\left(\left\|z_{2}+h_{2}\right\|-\left\|z_{2}\right\|\right) \operatorname{ctan} \theta\right)+O\left(\|h\|^{1+\rho}\right) \\
= & f\left(\lambda_{1}(z)\right)+f^{\prime}\left(\lambda_{1}(z+h)\right)\left(h_{1}-\frac{\left(z_{2}+h_{2}\right)^{T} h_{2}}{\left\|z_{2}+h_{2}\right\|} \operatorname{ctan} \theta\right) \\
& +O\left(\|h\|^{2}\right)+O\left(\|h\|^{1+\rho}\right) \\
= & f\left(\lambda_{1}(z)\right)+f^{\prime}\left(\lambda_{1}(z+h)\right)\left(h_{1}-\frac{\left(z_{2}+h_{2}\right)^{T} h_{2}}{\left\|z_{2}+h_{2}\right\|} \operatorname{ctan} \theta\right) \\
& +O\left(\|h\|^{1+\min \{1, \rho\}}\right),
\end{aligned}
$$

where the second equation is due to Lemma 3 and the last equality comes from the boundedness of $f^{\prime}$, since $f$ is strictly continuous at $\lambda_{1}(z)$. Similar argument holds for $f\left(\lambda_{2}(z+h)\right)$. Hence the first component of (54) is $O\left(\|h\|^{1+\min \{1, \rho\}}\right)$.

Next, let us look into the second component of (54), which involved $\lambda_{1}(z)$. By Lemma 1 again, it can be expressed as

$$
\begin{aligned}
& -\frac{\operatorname{ctan} \theta}{1+\operatorname{ctan}^{2} \theta} f\left(\lambda_{1}(z+h)\right) \frac{z_{2}+h_{2}}{\left\|z_{2}+h_{2}\right\|} \\
& +\frac{\operatorname{ctan} \theta}{1+\operatorname{ctan}^{2} \theta} f^{\prime}\left(\lambda_{1}(z+h) ; h_{1}-\frac{\left(z_{2}+h_{2}\right)^{T} h_{2}}{\left\|z_{2}+h_{2}\right\|} \operatorname{ctan} \theta\right) \\
& \quad \times \frac{z_{2}+h_{2}}{\left\|z_{2}+h_{2}\right\|} \\
& +\frac{\operatorname{ctan} \theta}{1+\operatorname{ctan}^{2} \theta} f\left(\lambda_{1}(z)\right) \frac{z_{2}}{\left\|z_{2}\right\|} \\
& \quad+\frac{\operatorname{ctan} \theta_{1+\operatorname{ctan}^{2} \theta}}{\frac{f\left(\lambda_{1}(z+h)\right)}{\left\|z_{2}+h_{2}\right\|} M_{\left(z_{2}+h_{2}\right)} h .}
\end{aligned}
$$

Note that $\Phi$ is continuous differentiable (and hence is semismooth) with $\nabla \Phi\left(z_{2}\right)=\left(1 /\left\|z_{2}\right\|\right)\left(I-\left(z_{2} z_{2}^{T} /\left\|z_{2}\right\|^{2}\right)\right)$ and $M_{\left(z_{2}+h_{2}\right)} h=\left\|z_{2}+h_{2}\right\| \nabla \Phi\left(z_{2}+h_{2}\right) h_{2}$. Thus, expression (58) can be rewritten as

$$
\begin{aligned}
& -\frac{\operatorname{ctan} \theta}{1+\operatorname{ctan}^{2} \theta} f\left(\lambda_{1}(z+h)\right) \Phi\left(z_{2}+h_{2}\right) \\
& +\frac{\operatorname{ctan} \theta}{1+\operatorname{ctan}^{2} \theta} f^{\prime}\left(\lambda_{1}(z+h) ; h_{1}-\frac{\left(z_{2}+h_{2}\right)^{T} h_{2}}{\left\|z_{2}+h_{2}\right\|} \operatorname{ctan} \theta\right) \\
& \times \Phi\left(z_{2}+h_{2}\right) \\
& +\frac{\operatorname{ctan} \theta}{1+\operatorname{ctan}^{2} \theta} f\left(\lambda_{1}(z)\right) \Phi\left(z_{2}\right) \\
& +\frac{\operatorname{ctan} \theta}{1+\operatorname{ctan}^{2} \theta} f\left(\lambda_{1}(z+h)\right) \nabla \Phi\left(z_{2}+h_{2}\right) h_{2} \\
& =\frac{\operatorname{ctan} \theta}{1+\operatorname{ctan}^{2} \theta} \\
& \times\left[-f\left(\lambda_{1}(z+h)\right)+f\left(\lambda_{1}(z)\right)\right. \\
& \left.+f^{\prime}\left(\lambda_{1}(z+h) ; h_{1}-\frac{\left(z_{2}+h_{2}\right)^{T} h_{2}}{\left\|z_{2}+h_{2}\right\|} \operatorname{ctan} \theta\right)\right] \\
& \times \Phi\left(z_{2}+h_{2}\right) \\
& +\frac{f\left(\lambda_{1}(z)\right) \operatorname{ctan} \theta}{1+\operatorname{ctan}^{2} \theta} \\
& \times\left[-\Phi\left(z_{2}+h_{2}\right)+\Phi\left(z_{2}\right)+\nabla \Phi\left(z_{2}+h_{2}\right) h_{2}\right] \\
& +\frac{\operatorname{ctan} \theta}{1+\operatorname{ctan}^{2} \theta} \nabla \Phi\left(z_{2}+h_{2}\right) h_{2} \\
& \times\left[f\left(\lambda_{1}(z+h)\right)-f\left(\lambda_{1}(z)\right)\right] \\
& =O\left(\|h\|^{1+\min \{1, \rho\}}\right)+O\left(\|h\|^{2}\right)+O\left(\|h\|^{2}\right) \\
& =O\left(\|h\|^{1+\min \{1, \rho\}}\right) \text {. }
\end{aligned}
$$

The second equation comes from (57), strongly semismoothness of $\Phi$ at $z_{2}$, and

$$
\left\|\nabla \Phi\left(z_{2}+h_{2}\right) h_{2}\left[f\left(\lambda_{1}(z+h)\right)-f\left(\lambda_{1}(z)\right)\right]\right\|=O\left(\|h\|^{2}\right),
$$

since $f$ is Lipschitz at $\lambda_{1}(z)$ (which is ensured by the $\rho$-order semismoothness of $f$ ). Analogous arguments apply for the second component of (54) involving $\lambda_{2}(z)$. From all the above, we may conclude that

$$
\begin{aligned}
f^{\mathscr{L}_{\theta}}(z+h)-f^{\mathscr{L}_{\theta}}(z)-\left(f^{\mathscr{L}_{\theta}}\right)^{\prime}(z+h ; h) \\
=O\left(\|h\|^{1+\min \{1, \rho\}}\right)
\end{aligned}
$$

which says $f^{\mathscr{L}_{\theta}}$ is $\min \{1, \rho\}$-order semismooth at $z$ under this case. 
Case 2. For $z_{2}=0$, if $h_{2}=0$, then the proof is trivial. If $h_{2} \neq 0$, then the first component of (54) satisfies

$$
\begin{aligned}
& \frac{1}{1+\operatorname{ctan}^{2} \theta} \\
& \times\left[f\left(\lambda_{1}(z+h)\right)-f\left(z_{1}\right),\right. \\
& \left.\quad-f^{\prime}\left(\lambda_{1}(z+h) ; h_{1}-\left\|h_{2}\right\| \operatorname{ctan} \theta\right)\right] \\
& +\frac{1}{1+\tan ^{2} \theta} \\
& \times\left[f\left(\lambda_{2}(z+h)\right)-f\left(z_{1}\right)\right. \\
& \left.\quad-f^{\prime}\left(\lambda_{2}(z+h) ; h_{1}+\left\|h_{2}\right\| \tan \theta\right)\right] \\
& =O\left(\left|h_{1}-\left\|h_{2}\right\| \operatorname{ctan} \theta\right|^{1+\rho}\right)+O\left(\left|h_{1}+\left\|h_{2}\right\| \tan \theta\right|^{1+\rho}\right) \\
& =O\left(\|h\|^{1+\rho}\right),
\end{aligned}
$$

because $f$ is $\rho$-order semismooth at $z_{1}$. The second component of (54), by letting $\bar{z}_{2}=\bar{h}_{2}$ and $M_{h_{2}} h=0$, takes the form

$$
\begin{aligned}
& -\frac{\operatorname{ctan} \theta}{1+\operatorname{ctan}^{2} \theta} f\left(\lambda_{1}(z+h)\right) \bar{h}_{2} \\
& \quad+\frac{\operatorname{ctan} \theta}{1+\operatorname{ctan}^{2} \theta} f^{\prime}\left(\lambda_{1}(z+h) ; h_{1}-\left\|h_{2}\right\| \operatorname{ctan} \theta\right) \bar{h}_{2} \\
& \quad+\frac{\tan \theta}{1+\tan ^{2} \theta} f\left(\lambda_{2}(z+h)\right) \bar{h}_{2} \\
& \quad-\frac{\tan \theta}{1+\tan ^{2} \theta} f^{\prime}\left(\lambda_{2}(z+h) ; h_{1}+\left\|h_{2}\right\| \tan \theta\right) \bar{h}_{2} \\
& =-\frac{1}{\tan \theta+\operatorname{ctan} \theta}\left[f\left(\lambda_{1}(z+h)\right)-f\left(z_{1}\right)\right. \\
& \quad-\frac{1}{\tan \theta+\operatorname{ctan} \theta}\left[f\left(\lambda_{2}(z+h)\right)-f\left(z_{1}\right)\right. \\
& \left.=O\left(\|h\|^{1+\rho}\right), \quad-f^{\prime}\left(\lambda_{2}(z+h) ; h_{1}+\left\|h_{2}\right\| \tan \theta\right)\right] \bar{h}_{2}
\end{aligned}
$$

where the last step is due to the $\rho$-order semismoothness of $f$.

(b) Suppose that $f^{\mathscr{L}_{\theta}}$ is $\rho$-order semismooth at $z$. Let $t \epsilon$ $\mathbb{R}$ such that $f$ is differentiable at $\lambda_{1}(z)+t$. We discuss the following two cases.

Case 1. For $z_{2} \neq 0$, from $f$ being Lipschitz at $\lambda_{2}(z)$ (and hence the differentiable points are dense near $\lambda_{2}(z)$ ), there exists $\beta(t) \in \mathbb{R}$ such that $\beta(t)=O\left(|t|^{1+\rho}\right)$ and $f$ is differentiable at $\lambda_{2}(z)+\beta(t)$ and $\lambda_{2}(z)+\beta(t)>\lambda_{1}(z)+t$ as $t$ is sufficiently small (since $\lambda_{2}(z)>\lambda_{1}(z)$ by $\left.z_{2} \neq 0\right)$. Denote $h:=t u_{z}^{1}+\beta(t) u_{z}^{2}$. Then, $z+h=\left[\lambda_{1}(z)+t\right] u_{z}^{1}+\left[\lambda_{2}(z)+\beta(t)\right] u_{z}^{2}$ which implies $\lambda_{1}(z+h)=\lambda_{1}(z)+t$ and $\lambda_{2}(z+h)=\lambda_{2}(z)+\beta(t)$. Since $f$ is differentiable at $\lambda_{1}(z)+t$ and $\lambda_{2}(z)+\beta(t), f^{\mathscr{L}_{\theta}}$ is also differentiable at $z+h$ by Lemma 2 . Notice that

$$
\begin{aligned}
& h=\left[\begin{array}{l}
h_{1} \\
h_{2}
\end{array}\right] \\
&=\left[\begin{array}{c}
\frac{1}{1+\operatorname{ctan}^{2} \theta} t+\frac{1}{1+\tan ^{2} \theta} \beta(t) \\
\left(-\frac{\operatorname{ctan} \theta}{1+\operatorname{ctan}^{2} \theta} t+\frac{\tan \theta}{1+\tan ^{2} \theta} \beta(t)\right) \frac{z_{2}}{\left\|z_{2}\right\|}
\end{array}\right], \\
& z_{2}+h_{2}=\left(\left\|z_{2}\right\|-\frac{\operatorname{ctan} \theta}{1+\operatorname{ctan}^{2} \theta} t+\frac{\tan \theta}{1+\tan ^{2} \theta} \beta(t)\right) \frac{z_{2}}{\left\|z_{2}\right\|} .
\end{aligned}
$$

Hence,

$$
\frac{\left(z_{2}+h_{2}\right)^{T} h_{2}}{\left\|z_{2}+h_{2}\right\|}=-\frac{\operatorname{ctan} \theta}{1+\operatorname{ctan}^{2} \theta} t+\frac{\tan \theta}{1+\tan ^{2} \theta} \beta(t),
$$

which follows from the fact that $\left\|z_{2}\right\| \neq 0$ and $t$ can be arbitrarily small (hence $\left\|z_{2}\right\|-\left(\operatorname{ctan} \theta /\left(1+\operatorname{ctan}^{2} \theta\right)\right) t+(\tan \theta /(1+$ $\left.\left.\left.\tan ^{2} \theta\right)\right) \beta(t)>0\right)$. Thus, it is clear that

$$
\begin{aligned}
h_{1}- & \frac{\left(z_{2}+h_{2}\right)^{T} h_{2}}{\left\|z_{2}+h_{2}\right\|} \operatorname{ctan} \theta \\
= & \left(\frac{1}{1+\operatorname{ctan}^{2} \theta} t+\frac{1}{1+\tan ^{2} \theta} \beta(t)\right) \\
& -\left(-\frac{\operatorname{ctan} \theta}{1+\operatorname{ctan}^{2} \theta} t+\frac{\tan \theta}{1+\tan ^{2} \theta} \beta(t)\right) \operatorname{ctan} \theta=t, \\
h_{1}+ & \frac{\left(z_{2}+h_{2}\right)^{T} h_{2}}{\left\|z_{2}+h_{2}\right\|} \tan \theta \\
= & \left(\frac{1}{1+\operatorname{ctan}^{2} \theta} t+\frac{1}{1+\tan ^{2} \theta} \beta(t)\right) \\
& +\left(-\frac{\operatorname{ctan}^{2} \theta}{1+\operatorname{ctan}^{2} \theta} t+\frac{\tan ^{2} \theta}{1+\tan ^{2} \theta} \beta(t)\right) \tan \theta=\beta(t) .
\end{aligned}
$$

In addition, it can be verified that

$$
\begin{aligned}
\left|h_{1}\right| & =\left|\frac{1}{1+\operatorname{ctan}^{2} \theta} t+\frac{1}{1+\tan ^{2} \theta} \beta(t)\right| \\
& \leq \frac{1}{1+\operatorname{ctan}^{2} \theta}|t|+\frac{1}{1+\tan ^{2} \theta}|t|=|t|,
\end{aligned}
$$

since $\beta(t)=O\left(|t|^{1+\rho}\right) \leq|t|$ as $t$ is sufficiently small. Similarly,

$$
\begin{aligned}
\left\|h_{2}\right\| & =\left\|\left[-\frac{\operatorname{ctan} \theta}{1+\operatorname{ctan}^{2} \theta} t+\frac{\tan \theta}{1+\tan ^{2} \theta} \beta(t)\right] \frac{z_{2}}{\left\|z_{2}\right\|}\right\| \\
& =\left|-\frac{\operatorname{ctan} \theta}{1+\operatorname{ctan}^{2} \theta} t+\frac{\tan \theta}{1+\tan ^{2} \theta} \beta(t)\right| \\
& \leq \frac{\operatorname{ctan} \theta}{1+\operatorname{ctan}^{2} \theta}|t|+\frac{\tan \theta}{1+\tan ^{2} \theta}|t| \leq|t| .
\end{aligned}
$$


Therefore, we obtain $\|h\|=O(t)$ which further implies $O\left(\|h\|^{1+\rho}\right)=O\left(|t|^{1+\rho}\right)$. Then, by the hypothesis $f^{\mathscr{L}_{\theta}}$ being $\rho$ order semismooth at $z$, that is,

$$
f^{\mathscr{L}_{\theta}}(z+h)-f^{\mathscr{L}_{\theta}}(z)-\left(f^{\mathscr{L}_{\theta}}\right)^{\prime}(z+h ; h)=O\left(\|h\|^{1+\rho}\right),
$$

we have

$$
\begin{aligned}
& \left\langle f^{\mathscr{L}_{\theta}}(z+h)-f^{\mathscr{L}_{\theta}}(z)-\left(f^{\mathscr{L}_{\theta}}\right)^{\prime}(z+h ; h), e\right\rangle \\
& =O\left(\|h\|^{1+\rho}\right)=O\left(|t|^{1+\rho}\right) .
\end{aligned}
$$

In fact, the left-hand side of (71) takes the form of

$$
\begin{aligned}
& \frac{f\left(\lambda_{1}(z+h)\right)}{1+\operatorname{ctan}^{2} \theta}+\frac{f\left(\lambda_{2}(z+h)\right)}{1+\tan ^{2} \theta}-\frac{f\left(\lambda_{1}(z)\right)}{1+\operatorname{ctan}^{2} \theta}-\frac{f\left(\lambda_{2}(z)\right)}{1+\tan ^{2} \theta} \\
& -\frac{f^{\prime}\left(\lambda_{1}(z+h)\right)}{1+\operatorname{ctan}^{2} \theta}\left(h_{1}-\frac{\left(z_{2}+h_{2}\right)^{T} h_{2}}{\left\|z_{2}+h_{2}\right\|} \operatorname{ctan} \theta\right) \\
& -\frac{f^{\prime}\left(\lambda_{2}(z+h)\right)}{1+\tan ^{2} \theta}\left(h_{1}+\frac{\left(z_{2}+h_{2}\right)^{T} h_{2}}{\left\|z_{2}+h_{2}\right\|} \tan \theta\right) \\
& =\frac{f\left(\lambda_{1}(z)+t\right)}{1+\operatorname{ctan}^{2} \theta}+\frac{f\left(\lambda_{2}(z)+\beta(t)\right)}{1+\tan ^{2} \theta} \\
& -\frac{f\left(\lambda_{1}(z)\right)}{1+\operatorname{ctan}^{2} \theta}-\frac{f\left(\lambda_{2}(z)\right)}{1+\tan ^{2} \theta} \\
& -\frac{f^{\prime}\left(\lambda_{1}(z)+t\right)}{1+\operatorname{ctan}^{2} \theta} t-\frac{f^{\prime}\left(\lambda_{2}(z)+\beta(t)\right)}{1+\tan ^{2} \theta} \beta(t) \\
& =\frac{1}{1+\operatorname{ctan}^{2} \theta} \\
& \times\left[f\left(\lambda_{1}(z)+t\right)-f\left(\lambda_{1}(z)\right)-f^{\prime}\left(\lambda_{1}(z)+t\right) t\right] \\
& +\frac{1}{1+\tan ^{2} \theta}\left[f\left(\lambda_{2}(z)+\beta(t)\right)-f\left(\lambda_{2}(z)\right)\right. \\
& \left.-f^{\prime}\left(\lambda_{2}(z)+\beta(t)\right) \beta(t)\right] \\
& =\frac{1}{1+\operatorname{ctan}^{2} \theta} \\
& \times\left[f\left(\lambda_{1}(z)+t\right)-f\left(\lambda_{1}(z)\right)-f^{\prime}\left(\lambda_{1}(z)+t\right) t\right] \\
& +O\left(|t|^{1+\rho}\right)
\end{aligned}
$$

where the last step is due to the fact that $f^{\prime}$ is bounded and

$$
f\left(\lambda_{2}(z)+\beta(t)\right)-f\left(\lambda_{2}(z)\right)=O\left(|t|^{1+\rho}\right),
$$

since $f$ is Lipschitz at $\lambda_{2}(z)$. Hence (71) means

$$
f\left(\lambda_{1}(z)+t\right)-f\left(\lambda_{1}(z)\right)-f^{\prime}\left(\lambda_{1}(z)+t\right) t=O\left(|t|^{1+\rho}\right),
$$

which says $f$ is $\rho$-order semismooth at $\lambda_{1}(z)$. Applying similar arguments show that $f$ is $\rho$-order semismooth at $\lambda_{2}(z)$.
Case 2. For $z_{2}=0$, letting $h=t e$. Since $f$ is differentiable at $\lambda_{1}(z)+t=z_{1}+t$ and $\lambda_{i}(z+h)=z_{1}+t$, for $i=1,2, f^{\mathscr{L}_{\theta}}$ is differentiable at $z+h$ by Lemma 2; that is, $z+h \in D_{f^{\mathscr{L}_{\theta}}}$. Because $f^{\mathscr{L}_{\theta}}$ is $\rho$-order semismooth at $z$, we have

$$
f^{\mathscr{L}_{\theta}}(z+h)-f^{\mathscr{L}_{\theta}}(z)-\left(f^{\mathscr{L}_{\theta}}\right)^{\prime}(z+h ; h)=O\left(\|h\|^{1+\rho}\right),
$$

which, together with the fact $\|h\|=|t|$, is equivalent to

$$
f\left(z_{1}+t\right)-f\left(z_{1}\right)-f^{\prime}\left(z_{1}+t\right) t=O\left(|t|^{1+\rho}\right) .
$$

This clearly proves that $f$ is $\rho$-order semismooth at $\lambda_{i}(z)=$ $z_{1}$, for $i=1,2$.

(c) The necessity comes from part (b), and the sufficiency follows from (62) and (63).

Corollary 10. Let $f: \mathbb{R} \rightarrow \mathbb{R}$ and $f^{\mathscr{L}_{\theta}}$ be defined as in (4). For $\rho \in(0,1], f^{\mathscr{L}_{\theta}}$ is $\rho$-order semismooth at $z$ if and only if $f$ is $\rho$-order semismooth at $\lambda_{i}(z)$, for $i=1,2$.

Remark 11. In the framework of second-order cone and positive semidefinite cone, the corresponding result to part (a) has been established; see [5, Proposition 7] and [6, Proposition 4.10]. In [17], the author study the $\rho$-order semismoothness of the spectral operator for $\rho \in(0,1]$. Here we further show that if $f^{\mathscr{L}_{\theta}}$ is $\rho$-semismooth, then $f$ is $\rho$-semismooth for all $\rho>0$. In addition, if $z_{2}=0$, then the $\rho$-order semismoothness of $f^{\mathscr{L}_{\theta}}$ and $f$ coincide with each other for all $\rho>0$. result.

Inspired by [5, Lemma 4], we also obtain the following

Theorem 12. Let $f: \mathbb{R} \rightarrow \mathbb{R}$ be strictly continuous. Then, for any $z \in \mathbb{R}^{n}$, the B-differential $\partial_{B}\left(f^{\mathscr{L}_{\theta}}\right)(z)$ is well defined and nonempty. Moreover,

(i) if $z_{2} \neq 0$, then

$$
\begin{aligned}
& \partial_{B}\left(f^{\mathscr{L}_{\theta}}\right)(z)=\left\{\begin{array}{cc}
\xi & \frac{\varrho z_{2}^{T}}{\left\|z_{2}\right\|} \\
\frac{\varrho z_{2}}{\left\|z_{2}\right\|} & a I+\frac{(\eta-a) z_{2} z_{2}^{T}}{\left\|z_{2}\right\|^{2}}
\end{array}\right] \mid \\
& a=\frac{f\left(\lambda_{2}(z)\right)-f\left(\lambda_{1}(z)\right)}{\lambda_{2}(z)-\lambda_{1}(z)}, \\
& \xi-\varrho \operatorname{ctan} \theta \in \partial_{B} f\left(\lambda_{1}(z)\right), \\
& \xi+\varrho \tan \theta \in \partial_{B} f\left(\lambda_{2}(z)\right),
\end{aligned}
$$

$$
\eta=\xi-\varrho(\operatorname{ctan} \theta-\tan \theta)\}
$$


(ii) if $z_{2}=0$, then

$$
\begin{array}{r}
\partial_{B}\left(f^{\mathscr{L}_{\theta}}\right)(z) \subset\left\{\left[\begin{array}{cc}
\xi & \varrho w^{T} \\
\varrho w & a I+(\eta-a) w w^{T}
\end{array}\right] \mid\right. \\
a \in \partial f\left(\lambda_{1}(z)\right),\|w\|=1, \\
\xi-\varrho \operatorname{ctan} \theta \in \partial_{B} f\left(\lambda_{1}(z)\right), \\
\xi+\varrho \tan \theta \in \partial_{B} f\left(\lambda_{1}(z)\right), \\
\eta=\xi-\varrho(\operatorname{ctan} \theta-\tan \theta)\} .
\end{array}
$$

Proof. Denote by $\Omega$ the set in the right side of (77).

Case 1. $z_{2} \neq 0$. For any sequence $\left\{z^{k}\right\}_{k=1}^{\infty} \rightarrow z$ with $z^{k} \in D_{\left(f^{\left.\mathscr{L}_{\theta}\right)}\right.}$. Then, we compute

$$
\begin{aligned}
& \nabla f^{\mathscr{L}_{\theta}}\left(z^{k}\right) \\
& =\left\{\begin{array}{cc}
{\left[\begin{array}{cc}
\xi\left(z^{k}\right) & \varrho\left(z^{k}\right)\left(\frac{z_{2}^{k}}{\left\|z_{2}^{k}\right\|}\right)^{T} \\
\frac{\varrho\left(z^{k}\right) z_{2}^{k}}{\left\|z_{2}^{k}\right\|} & a\left(z^{k}\right) I+\left(\eta\left(z^{k}\right)-a\left(z^{k}\right)\right) \frac{z_{2}^{k}\left(z^{k}\right)_{2}^{T}}{\left\|z_{2}^{k}\right\|^{2}}
\end{array}\right] \text { । }} \\
\eta\left(z^{k}\right)=\xi\left(z^{k}\right)-\varrho\left(z^{k}\right)(\operatorname{ctan} \theta-\tan \theta)
\end{array}\right\} .
\end{aligned}
$$

Since $f$ is strictly continuous, we know that $\xi\left(z^{k}\right)$ and $\varrho\left(z^{k}\right)$ are bounded and hence have cluster points. We assume, without loss of generality, that $\xi\left(z^{k}\right) \rightarrow \widetilde{\xi}$ and $\varrho\left(z^{k}\right) \rightarrow \widetilde{\varrho}$. Note that $f$ is differentiable at $\lambda_{i}\left(z^{k}\right)$ for $i=1,2$ by Lemma 2 . Besides, from

$$
\begin{aligned}
& \xi\left(z^{k}\right)-\varrho\left(z^{k}\right) \operatorname{ctan} \theta=f^{\prime}\left(\lambda_{1}\left(z^{k}\right)\right), \\
& \xi\left(z^{k}\right)+\varrho\left(z^{k}\right) \tan \theta=f^{\prime}\left(\lambda_{2}\left(z^{k}\right)\right),
\end{aligned}
$$

and the fact that any cluster point of $\left\{f^{\prime}\left(\lambda_{i}\left(z^{k}\right)\right)\right\}_{k=1}^{\infty}$ is in $\partial_{B} f\left(\lambda_{i}(z)\right)$ by definition, we have

$$
\widetilde{\xi}-\widetilde{\varrho} \operatorname{ctan} \theta \in \partial_{B} f\left(\lambda_{1}(z)\right), \quad \tilde{\xi}+\widetilde{\varrho} \tan \theta \in \partial_{B} f\left(\lambda_{2}(z)\right) .
$$

This means that any cluster points of $\left\{\nabla\left(f^{\mathscr{L}_{\theta}}\right)\left(z^{k}\right)\right\}_{k=1}^{\infty}$ are element of $\Omega$; that is, $\partial_{B}\left(f^{\mathscr{L}_{\theta}}\right)(z) \subset \Omega$.

Conversely, for any $\xi$ and $\varrho$ satisfying $\xi-\varrho \operatorname{ctan} \theta \in$ $\partial_{B} f\left(\lambda_{1}(z)\right)$ and $\xi+\varrho \tan \theta \in \partial_{B} f\left(\lambda_{2}(z)\right)$, there exist $\left\{\lambda_{1}^{k}\right\}_{k=1}^{\infty} \rightarrow$ $\lambda_{1}(z)$ and $\left\{\lambda_{2}^{k}\right\}_{k=1}^{\infty} \rightarrow \lambda_{2}(z)$ with $f$ being differentiable at $\lambda_{1}^{k}$ and $\lambda_{2}^{k}$ and $\left\{f^{\prime}\left(\lambda_{1}^{k}\right)\right\}_{k=1}^{\infty} \rightarrow \xi-\varrho \operatorname{ctan} \theta$ and $\left\{f^{\prime}\left(\lambda_{2}^{k}\right)\right\}_{k=1}^{\infty} \rightarrow \xi+$ $\varrho \tan \theta$. Since $\lambda_{2}(z)=z_{1}+\left\|z_{2}\right\| \tan \theta>z_{1}>z_{1}-\left\|z_{2}\right\| \operatorname{ctan} \theta=$ $\lambda_{1}(z)$, it implies that $\lambda_{2}^{k}>\lambda_{1}^{k}$ is $k$ large enough. Now, let

$$
z^{k}:=\left[\begin{array}{l}
z_{1}^{k} \\
z_{2}^{k}
\end{array}\right]=\left[\begin{array}{c}
\frac{\tan ^{2} \theta}{1+\tan ^{2} \theta} \lambda_{1}^{k}+\frac{\operatorname{ctan}^{2} \theta}{1+\operatorname{ctan}^{2} \theta} \lambda_{2}^{k} \\
\left(\frac{\tan \theta}{1+\tan ^{2} \theta} \lambda_{2}^{k}-\frac{\operatorname{ctan} \theta}{1+\operatorname{ctan}^{2} \theta} \lambda_{1}^{k}\right) \frac{z_{2}}{\left\|z_{2}\right\|}
\end{array}\right] .
$$

For points $z^{k}$, it is easy to see that $\lambda_{i}\left(z^{k}\right)=\lambda_{i}^{k}$ for all $k$ by using the following facts:

$$
\begin{aligned}
\frac{\tan \theta}{1+\tan ^{2} \theta} \lambda_{2}^{k} & =\frac{1}{\operatorname{ctan} \theta+\tan \theta} \lambda_{2}^{k}>\frac{1}{\operatorname{ctan} \theta+\tan \theta} \lambda_{1}^{k} \\
& =\frac{\operatorname{ctan} \theta}{1+\operatorname{ctan}^{2} \theta} \lambda_{1}^{k} .
\end{aligned}
$$

Hence, $z^{k} \rightarrow z$ and $f^{\mathscr{L}_{\theta}}$ are differentiable at $z^{k}$ by Lemma 2 (since $f$ is differentiable at $\lambda_{i}\left(z^{k}\right)=\lambda_{i}^{k}$ for $\left.i=1,2\right)$. Then, we compute

$$
\begin{gathered}
\nabla f^{\mathscr{L}_{\theta}}\left(z^{k}\right) \\
=\left\{\begin{array}{cc}
{\left[\begin{array}{cc}
\xi\left(z^{k}\right) & \varrho\left(z^{k}\right)\left(\frac{z_{2}}{\left\|z_{2}\right\|}\right)^{T} \\
\frac{\varrho\left(z^{k}\right) z_{2}}{\left\|z_{2}\right\|} & a\left(z^{k}\right) I+\left(\eta\left(z^{k}\right)-a\left(z^{k}\right)\right) \frac{z_{2} z_{2}^{T}}{\left\|z_{2}\right\|^{2}}
\end{array}\right] \mid} \\
\eta\left(z^{k}\right)=\xi\left(z^{k}\right)-\varrho\left(z^{k}\right)(\operatorname{ctan} \theta-\tan \theta)
\end{array}\right\},
\end{gathered}
$$

where

$$
\begin{aligned}
a\left(z^{k}\right) & =\frac{f\left(\lambda_{2}\left(z^{k}\right)\right)-f\left(\lambda_{1}\left(z^{k}\right)\right)}{\lambda_{2}\left(z^{k}\right)-\lambda_{1}\left(z^{k}\right)} \\
& \longrightarrow \frac{f\left(\lambda_{2}(z)\right)-f\left(\lambda_{1}(z)\right)}{\lambda_{2}(z)-\lambda_{1}(z)}=a, \\
\xi\left(z^{k}\right)= & \frac{f^{\prime}\left(\lambda_{1}^{k}\right)}{1+\operatorname{ctan}^{2} \theta}+\frac{f^{\prime}\left(\lambda_{2}^{k}\right)}{1+\tan ^{2} \theta} \\
& \longrightarrow \frac{\xi-\varrho \operatorname{ctan} \theta}{1+\operatorname{ctan}^{2} \theta}+\frac{\xi+\varrho \tan \theta}{1+\tan ^{2} \theta}=\xi, \\
\varrho\left(z^{k}\right)= & -\frac{\operatorname{ctan} \theta}{1+\operatorname{ctan}^{2} \theta} f^{\prime}\left(\lambda_{1}^{k}\right)+\frac{\tan \theta}{1+\tan ^{2} \theta} f^{\prime}\left(\lambda_{2}^{k}\right) \\
& \longrightarrow-\frac{\operatorname{ctan}^{k} \theta}{1+\operatorname{ctan}^{2} \theta}(\xi-\varrho \operatorname{ctan} \theta) \\
& +\frac{\tan ^{2} \theta}{1+\tan ^{2} \theta}(\xi+\varrho \tan \theta)=\varrho .
\end{aligned}
$$

Since the limit of $\left\{\nabla f^{\mathscr{L}_{\theta}}\left(z^{k}\right)\right\}_{k=1}^{\infty}$ is an element of $\partial_{B} f^{\mathscr{L}_{\theta}}(z)$, we obtain $\Omega \subset \partial_{B} f^{\mathscr{L}_{\theta}}(z)$. 
Case 2. $z_{2}=0$. Consider any sequence $\left\{z^{k}\right\}_{k=1}^{\infty}=$ $\left\{\left(z_{1}^{k}, z_{2}^{k}\right)\right\}_{k=1}^{\infty} \rightarrow z$ with $f^{\mathscr{L}_{\theta}}$ being differentiable at $z^{k}$ for all $k$. By passing to a subsequence, we can assume that either $z_{2}^{k}=$ 0 for all $k$ or $z_{2}^{k} \neq 0$ for all $k$. If $z_{2}^{k}=0$, then by Lemma 2 we know that $f$ is differentiable at $\lambda_{i}\left(z^{k}\right)=z_{1}^{k}$ for $i=$ 1,2 and $\nabla f^{\mathscr{L}_{\theta}}\left(z^{k}\right)=f^{\prime}\left(z_{1}^{k}\right) I$. Hence, the cluster point of $\left\{\nabla f^{\mathscr{L}_{\theta}}\left(z^{k}\right)\right\}_{k=1}^{\infty}$ is an element of (78) with $\varrho=0$ and $\xi=a \epsilon$ $\partial_{B} f\left(z_{1}\right) \subset \partial f\left(z_{1}\right)$. If $z_{2}^{k} \neq 0$, by passing to a subsequence we can assume without loss of generality that $\left\{z_{2}^{k} /\left\|z_{2}^{k}\right\|\right\} \rightarrow w$ for some $w$ with $\|w\|=1$. Note that

$$
\begin{aligned}
& \nabla f^{\mathscr{L}_{\theta}}\left(z^{k}\right) \\
& =\left\{\begin{array}{cc}
{\left[\begin{array}{cc}
\xi\left(z^{k}\right) & \varrho\left(z^{k}\right)\left(\frac{z_{2}^{k}}{\left\|z_{2}^{k}\right\|}\right)^{T} \\
\frac{\varrho\left(z^{k}\right) z_{2}^{k}}{\left\|z_{2}^{k}\right\|} & a\left(z^{k}\right) I+\left(\eta\left(z^{k}\right)-a\left(z^{k}\right)\right) \frac{z_{2} z_{2}^{T}}{\left\|z_{2}\right\|^{2}}
\end{array}\right] \mid} \\
\eta\left(z^{k}\right)=\xi\left(z^{k}\right)-\left(z^{k}\right)(\operatorname{ctan} \theta-\tan \theta)
\end{array}\right\} .
\end{aligned}
$$

Moreover, from $\xi\left(z^{k}\right)$ and $\varrho\left(z^{k}\right)$ being bounded (due to the strictly continuous of $f$ ), we can assume that $\xi\left(z^{k}\right) \rightarrow$ $\tilde{\xi}$ and $\varrho\left(z^{k}\right) \rightarrow \tilde{\varrho}$. Using (80) and any cluster point of $\left\{f^{\prime}\left(\lambda_{i}\left(z^{k}\right)\right)\right\}_{k=1}^{\infty}$ in $\partial_{B} f\left(\lambda_{i}(z)\right)$, we have

$$
\widetilde{\xi}-\tilde{\varrho} \operatorname{ctan} \theta \in \partial_{B} f\left(\lambda_{1}(z)\right), \quad \tilde{\xi}+\widetilde{\varrho} \tan \theta \in \partial_{B} f\left(\lambda_{2}(z)\right) \text {. }
$$

In addition,

$$
a\left(z^{k}\right)=\frac{f\left(\lambda_{2}\left(z^{k}\right)\right)-f\left(\lambda_{1}\left(z^{k}\right)\right)}{\lambda_{2}\left(z^{k}\right)-\lambda_{1}\left(z^{k}\right)} \in \partial f\left(\hat{\lambda}^{k}\right),
$$

where $\hat{\lambda}^{k} \in\left[\lambda_{1}\left(z^{k}\right), \lambda_{2}\left(z^{k}\right)\right]$ and hence converges to $\lambda_{1}(z)$, since $\lambda_{i}\left(z^{k}\right) \rightarrow \lambda_{1}(z)$ for $i=1,2$, due to $z_{2}=0$. Using the outer semicontinuity of $\partial f$ we get that the cluster point of $\left\{a\left(z^{k}\right)\right\}$ belongs to $\partial f\left(\lambda_{1}(z)\right)$. Hence any cluster of $\left\{\nabla f^{\mathscr{L}_{\theta}}\left(z^{k}\right)\right\}_{k=1}^{\infty}$ belongs to an element of the set of the right side in (78).

We point out one thing for Theorem 12(ii). In the set of the right side in (78), $a \in \partial f\left(\lambda_{1}(z)\right)$ cannot be replaced by $a \in$ $\partial_{B} f\left(\lambda_{1}(z)\right)$ because $\partial_{B} f\left(\lambda_{1}(z)\right)$ is usually a smaller subset of $\partial f\left(\lambda_{1}(z)\right)$. For example, letting $f(t)=|t|$ and $\alpha=2, \beta=-1$, we have

$$
\frac{f(\alpha)-f(\beta)}{\alpha-\beta}=\frac{1}{3} \notin \partial_{B} f(t) \quad \forall t \in \mathbb{R},
$$

while

$$
\frac{f(\alpha)-f(\beta)}{\alpha-\beta}=\frac{1}{3} \in \partial f(0)=[-1,1],
$$

which is the main reason causing what we just pointed out.
At present, we roughly describe $\partial_{B}\left(f^{\mathscr{L}_{\theta}}\right)$ for $z_{2}=0$. In other words, how to get the exact formula on $\partial_{B}\left(f^{\mathscr{L}_{\theta}}\right)$. Toward this end, we need to introduce the following definition. Given $z \in \mathbb{R}^{n}$, define

$\Gamma(z)$

$$
:=\lim _{\substack{t_{k} \rightarrow \lambda_{1}(z), t_{k}^{\prime} \rightarrow \lambda_{2}(z) \\ t_{k}, t_{k}^{\prime} \in D_{f}, t_{k}<t_{k}^{\prime}}}\left(f^{\prime}\left(t_{k}\right), f^{\prime}\left(t_{k}^{\prime}\right), \frac{f\left(t_{k}^{\prime}\right)-f\left(t_{k}\right)}{t_{k}^{\prime}-t_{k}}\right),
$$

where "lim sup" is the outer limits in the sense of set-valued mapping; see $[18,19]$ for more details.

First, for $z_{2} \neq 0$, according to Lemma 2 let us write the gradient of $\nabla f^{\mathscr{L}_{\theta}}$ as

$$
\begin{aligned}
& \nabla f^{\mathscr{L}_{\theta}}(z) \\
& =\frac{1}{\tan \theta+\operatorname{ctan} \theta} \\
& \times\left[\begin{array}{cc}
\tan \theta & -\left(\frac{z_{2}}{\left\|z_{2}\right\|}\right) \\
\frac{-z_{2}}{\left\|z_{2}\right\|} & \operatorname{ctan} \theta \frac{z_{2}\left(z_{2}\right)^{T}}{\left\|z_{2}\right\|^{2}}
\end{array}\right] f^{\prime}\left(\lambda_{1}(z)\right) \\
& +\frac{1}{\tan \theta+\operatorname{ctan} \theta}\left[\begin{array}{cc}
\operatorname{ctan} \theta & \left(\frac{z_{2}}{\left\|z_{2}\right\|}\right)^{T} \\
\frac{z_{2}}{\left\|z_{2}\right\|} & \tan \theta \frac{z_{2}\left(z_{2}\right)^{T}}{\left\|z_{2}\right\|^{2}}
\end{array}\right] f^{\prime}\left(\lambda_{2}(z)\right) \\
& +\frac{f\left(\lambda_{2}(z)\right)-f\left(\lambda_{1}(z)\right)}{\lambda_{2}(z)-\lambda_{1}(z)}\left[\begin{array}{cc}
0 & 0 \\
0 & I-\frac{z_{2}\left(z_{2}\right)^{T}}{\left\|z_{2}\right\|^{2}}
\end{array}\right] \text {. }
\end{aligned}
$$
below.

The exact formula of $B$-subdifferential $\partial_{B}\left(f^{\mathscr{L}_{\theta}}\right)$ is given

Theorem 13. Given $z \in \mathbb{R}^{n}$, the following statements hold.

(a) If $z_{2} \neq 0$, then

$$
\begin{gathered}
\partial_{B}\left(f^{\mathscr{L}_{\theta}}\right)(z) \\
=\left\{\frac{1}{\tan \theta+\operatorname{ctan} \theta}\left[\begin{array}{cc}
\tan \theta & -\bar{z}_{2} \\
-\bar{z}_{2} & \operatorname{ctan} \theta \bar{z}_{2} \bar{z}_{2}^{T}
\end{array}\right] \beta\right. \\
+\frac{1}{\tan \theta+\operatorname{ctan} \theta}\left[\begin{array}{cc}
\operatorname{ctan} \theta & \bar{z}_{2}^{T} \\
\bar{z}_{2} & \tan \theta \bar{z}_{2} \bar{z}_{2}^{T}
\end{array}\right] \gamma \\
\left.+\left[\begin{array}{ll}
0 & 0 \\
0 & I-\bar{z}_{2} \bar{z}_{2}^{T}
\end{array}\right] \varsigma \mid(\beta, \gamma, \varsigma) \in \Gamma(z)\right\} .
\end{gathered}
$$


(b) If $z_{2}=0$, then

$$
\begin{aligned}
& \partial_{B}\left(f^{\mathscr{L}_{\theta}}\right)(z)\left\{\frac{1}{\tan \theta+\operatorname{ctan} \theta}\left[\begin{array}{cc}
\tan \theta & -w^{T} \\
-w & \operatorname{ctan} \theta w w^{T}
\end{array}\right] \beta\right. \\
&+\frac{1}{\tan \theta+\operatorname{ctan} \theta}\left[\begin{array}{cc}
\operatorname{ctan} \theta & w^{T} \\
w & \tan \theta w w^{T}
\end{array}\right] \gamma \\
&\left.+\left[\begin{array}{ll}
0 & 0 \\
0 & I-w w^{T}
\end{array}\right] \varsigma \mid \begin{array}{r}
(\beta, \gamma, \varsigma) \in \Gamma(z) \\
\|w\|=1
\end{array}\right\} \\
& \cup\left\{\tau I \mid \tau \in \partial_{B} f\left(z_{1}\right)\right\} .
\end{aligned}
$$

Proof. (a) Denote by $\Xi$ the set in the right side of (93). Take $V \in \partial_{B}\left(f^{\mathscr{L}_{\theta}}\right)(z)$. By definition, there exists a sequence $\left\{z^{k}\right\}_{k=1}^{\infty} \rightarrow z$ with $z^{k} \in D_{\left(f^{\left.\mathscr{L}_{\theta}\right)}\right.}$ satisfying $\nabla f^{\mathscr{L}_{\theta}}\left(z^{k}\right) \rightarrow V$. Since $z_{2} \neq 0$, then $z_{2}^{k} \neq 0$ for $k$ sufficiently large. Note that $\lambda_{i}\left(z^{k}\right) \in D_{f}$ for $i=1,2$ by Lemma 2 and from (92)

$$
\begin{aligned}
& \nabla f^{\mathscr{L}_{\theta}}\left(z^{k}\right)=\frac{1}{\tan \theta+\operatorname{ctan} \theta} \\
& \times\left[\begin{array}{cc}
\tan \theta & -\left(\frac{z_{2}^{k}}{\left\|z_{2}^{k}\right\|}\right) \\
\frac{-z_{2}^{k}}{\left\|z_{2}^{k}\right\|} & \operatorname{ctan} \theta \frac{z_{2}^{k}\left(z_{2}^{k}\right)^{T}}{\left\|z_{2}^{k}\right\|^{2}}
\end{array}\right] f^{\prime}\left(\lambda_{1}\left(z^{k}\right)\right) \\
& +\frac{1}{\tan \theta+\operatorname{ctan} \theta} \\
& \times\left[\begin{array}{cc}
\operatorname{ctan} \theta & \left(\frac{z_{2}^{k}}{\left\|z_{2}^{k}\right\|}\right)^{T} \\
\frac{z_{2}^{k}}{\left\|z_{2}^{k}\right\|} & \tan \theta \frac{z_{2}^{k}\left(z_{2}^{k}\right)^{T}}{\left\|z_{2}^{k}\right\|^{2}}
\end{array}\right] f^{\prime}\left(\lambda_{2}\left(z^{k}\right)\right) \\
& +\frac{f\left(\lambda_{2}\left(z^{k}\right)\right)-f\left(\lambda_{1}\left(z^{k}\right)\right)}{\lambda_{2}\left(z^{k}\right)-\lambda_{1}\left(z^{k}\right)} \\
& \times\left[\begin{array}{cc}
0 & 0 \\
0 & I-\frac{z_{2}^{k}\left(z_{2}^{k}\right)^{T}}{\left\|z_{2}^{k}\right\|^{2}}
\end{array}\right] .
\end{aligned}
$$

Note also that $z_{2}^{k} /\left\|z_{2}^{k}\right\| \rightarrow z_{2} /\left\|z_{2}\right\|=\bar{z}_{2}, \lambda_{i}\left(z^{k}\right) \rightarrow \lambda_{i}(z)$, for $i=1,2$, and

$$
\begin{aligned}
& \left(f^{\prime}\left(\lambda_{1}\left(z^{k}\right)\right), f^{\prime}\left(\lambda_{2}\left(z^{k}\right)\right), \frac{f\left(\lambda_{2}\left(z^{k}\right)\right)-f\left(\lambda_{1}\left(z^{k}\right)\right)}{\lambda_{2}\left(z^{k}\right)-\lambda_{1}\left(z^{k}\right)}\right) \\
& \quad \longrightarrow \Gamma(z) .
\end{aligned}
$$

Hence $V=\lim _{k \rightarrow \infty} \nabla f^{\mathscr{L}_{\theta}}\left(z^{k}\right) \in \Xi$. This establishes $\partial_{B}\left(f^{\mathscr{L}_{\theta}}\right)(z) \subset$ $\Xi$.
Conversely, take $V \in \Xi$; that is, there exists $(\beta, \gamma, \varsigma) \in \Gamma(z)$ such that

$$
\begin{aligned}
V= & \frac{1}{\tan \theta+\operatorname{ctan} \theta}\left[\begin{array}{cc}
\tan \theta & -\bar{z}_{2} \\
-\bar{z}_{2} & \operatorname{ctan} \theta \bar{z}_{2} \bar{z}_{2}^{T}
\end{array}\right] \beta \\
& +\frac{1}{\tan \theta+\operatorname{ctan} \theta}\left[\begin{array}{cc}
\operatorname{ctan} \theta & \bar{z}_{2}^{T} \\
\bar{z}_{2} & \tan \theta \bar{z}_{2} \bar{z}_{2}^{T}
\end{array}\right] \gamma \\
& +\left[\begin{array}{cc}
0 & 0 \\
0 & I-\bar{z}_{2} \bar{z}_{2}^{T}
\end{array}\right] \varsigma .
\end{aligned}
$$

By definition of $\Gamma(z)$, there exists $t_{k}, t_{k}^{\prime} \in D_{f}$ such that $t_{k} \rightarrow$ $\lambda_{1}(z), t_{k}^{\prime} \rightarrow \lambda_{2}(z), t_{k}^{\prime}>t_{k}$, and

$$
\left(f^{\prime}\left(t_{k}\right), f^{\prime}\left(t_{k}^{\prime}\right), \frac{f\left(t_{k}^{\prime}\right)-f\left(t_{k}\right)}{t_{k}^{\prime}-t_{k}}\right) \longrightarrow(\beta, \gamma, \varsigma) .
$$

Let

$$
z^{k}:=\left[\begin{array}{l}
z_{1}^{k} \\
z_{2}^{k}
\end{array}\right]=\left[\begin{array}{c}
\frac{\tan ^{2} \theta}{1+\tan ^{2} \theta} t_{k}+\frac{\operatorname{ctan}^{2} \theta}{1+\operatorname{ctan}^{2} \theta} t_{k}^{\prime} \\
\left(\frac{\tan \theta}{1+\tan ^{2} \theta} t_{k}^{\prime}-\frac{\operatorname{ctan} \theta}{1+\operatorname{ctan}^{2} \theta} t_{k}\right) \frac{z_{2}}{\left\|z_{2}\right\|}
\end{array}\right] .
$$

Note that $t_{k}^{\prime}>t_{k}$, it is easy to see the $\lambda_{1}\left(z^{k}\right)=t_{k}, \lambda_{2}\left(z_{k}\right)=$ $t_{k}^{\prime}$ and $u_{z^{k}}^{i}=u_{z}^{i}$ for $i=1,2$. Hence, $z^{k} \rightarrow z$ and $f^{\mathscr{L}_{\theta}}$ are differentiable at $z^{k}$ by Lemma 2 (since $f$ is differentiable at $\lambda_{i}\left(z^{k}\right)$ for $\left.i=1,2\right)$. Hence according to the formula of gradients $\nabla f^{\mathscr{L}_{\theta}}\left(z^{k}\right),(95),(97)$, and (98), together with the fact $z_{2}^{k} /\left\|z_{2}^{k}\right\|=z_{2} /\left\|z_{2}\right\|$, we have $V=\lim _{k \rightarrow \infty} \nabla f^{\mathscr{L}_{\theta}}\left(z^{k}\right)$, which in turn implies $V \in \partial_{B}\left(f^{\mathscr{L}_{\theta}}\right)(z)$.

(b) Take $V \in \partial_{B}\left(f^{\mathscr{L}_{\theta}}\right)(z)$; then by definition there exists $z^{k} \rightarrow z$ with $z^{k} \in D_{f^{\mathscr{L}_{\theta}}}$ such that $\nabla f^{\mathscr{L}_{\theta}}\left(z^{k}\right) \rightarrow V$. By passing to a subsequence, we can assume that either $z_{2}^{k}=0$ for all $k$ or $z_{2}^{k} \neq 0$ for all $k$. If $z_{2}^{k}=0$, then by Lemma 2 $\nabla f^{\mathscr{L}_{\theta}}\left(z^{k}\right)=f^{\prime}\left(z_{1}^{k}\right) I$. Hence the cluster point of $\left\{f^{\prime}\left(z_{1}^{k}\right) I\right\}$ is an element of $\left\{\tau I \mid \tau \in \partial_{B} f\left(z_{1}\right)\right\}$. If $z_{2}^{k} \neq 0$, by passing to a subsequence we can assume that $\left\{z_{2}^{k} /\left\|z_{2}^{k}\right\|\right\} \rightarrow w$ for some $w$ with $\|w\|=1$. Note that $\lambda_{i}\left(z^{k}\right) \in D_{f}$ (since $z^{k} \in D_{f^{\mathscr{L}_{\theta}}}$ by Lemma 2), $\lambda_{i}\left(z^{k}\right) \rightarrow \lambda_{i}(z)$, for $i=1,2$, and $\lambda_{1}\left(z^{k}\right)<\lambda_{2}\left(z^{k}\right)$; it follows from (95) that $V$ belongs to an element of (94) with

$$
\begin{array}{r}
(\beta, \gamma, \varsigma) \\
\underset{\lim \sup _{k \rightarrow \infty}}{ }\left(f^{\prime}\left(\lambda_{1}\left(z^{k}\right)\right), f^{\prime}\left(\lambda_{2}\left(z^{k}\right)\right),\right. \\
\left.\frac{f\left(\lambda_{2}\left(z^{k}\right)\right)-f\left(\lambda_{1}\left(z^{k}\right)\right)}{\lambda_{2}\left(z^{k}\right)-\lambda_{1}\left(z^{k}\right)}\right) .
\end{array}
$$


Conversely, take $V$ belonging to the left side of (94); that is, there exists $\tau \in \partial_{B} f\left(z_{1}\right)$ such that $V=\tau I$ or exist $(\beta, \gamma, \varsigma) \in$ $\Gamma(z)$ and $w \in \mathbb{R}^{n-1}$ with $\|w\|=1$ such that

$$
\begin{aligned}
V= & \frac{1}{\tan \theta+\operatorname{ctan} \theta}\left[\begin{array}{cc}
\tan \theta & -w^{T} \\
-w & \operatorname{ctan} \theta w w^{T}
\end{array}\right] \beta \\
& +\frac{1}{\tan \theta+\operatorname{ctan} \theta}\left[\begin{array}{cc}
\operatorname{ctan} \theta & w^{T} \\
w & \tan \theta w w^{T}
\end{array}\right] \gamma \\
& +\left[\begin{array}{cc}
0 & 0 \\
0 & I-w w^{T}
\end{array}\right] \varsigma .
\end{aligned}
$$

If $V=\tau I$ for some $\tau \in \partial_{B} f\left(z_{1}\right)$, then there exists $t^{k} \rightarrow z_{1}$ with $t^{k} \in D_{f}$ such that $f^{\prime}\left(t^{k}\right) \rightarrow \tau$. Let $z^{k}=t^{k} e$, then $\lambda_{i}\left(z^{k}\right)=$ $t^{k}$, for $i=1,2, z^{k} \rightarrow z$, and $z^{k} \in D_{f^{\mathscr{L}_{\theta}}}$ by Lemma 2 . Thus, $\nabla f\left(z^{k}\right)=f^{\prime}\left(t^{k}\right) I$, which further implies $V=\tau I=$ $\lim _{k \rightarrow \infty} f^{\prime}\left(t^{k}\right) I=\lim _{k \rightarrow \infty} \nabla f^{\mathscr{L}_{\theta}}\left(z^{k}\right)$; that is, $V \in \partial_{B}\left(f^{\mathscr{L}_{\theta}}\right)(z)$. The remaining case can be proved by using the same argument following (97) by replacing $\bar{z}_{2}$ by $w$. The proof is complete.

Due to the important role played by $\Gamma(z)$, we present the estimate of $\Gamma$ as below.

Lemma 14. Given $z \in \mathbb{R}^{n}$, the following statements hold.

(a) If $z_{2} \neq 0$, then

$\Gamma(z)$

$$
=\left(\partial_{B} f\left(\lambda_{1}(z)\right), \partial_{B} f\left(\lambda_{2}(z)\right), \frac{f\left(\lambda_{2}(z)\right)-f\left(\lambda_{1}(z)\right)}{\lambda_{2}(z)-\lambda_{1}(z)}\right) .
$$

(b) If $z_{2}=0$, then

$$
\Gamma(z) \subset\left(\partial_{B} f\left(z_{1}\right), \partial_{B} f\left(z_{1}\right), \partial f\left(z_{1}\right)\right) .
$$

Proof. The case of $z_{2} \neq 0$ is clear, while the case of $z_{2}=0$ can be proved by using the similar argument following (88).

The exact estimate of $\Gamma(z)$ at $z_{2}=0$ can be obtained provided that additional assumption is imposed on $f$.

Lemma 15. Suppose that $f: \mathbb{R} \rightarrow \mathbb{R}$ is strictly continuous and directionally differentiable function satisfying

$$
\begin{aligned}
\lim _{\substack{t, v \rightarrow \tau^{\sigma} \\
t \neq v}} \frac{f(t)-f(v)}{t-v} & =\lim _{\substack{t \rightarrow \tau^{\sigma} \\
t \in D_{f}}} f^{\prime}(t)=f_{\sigma}^{\prime}(\tau), \\
& \forall \tau \in \mathbb{R}, \quad \sigma \in\{-,+\} .
\end{aligned}
$$

Then, for $z=\left(z_{1}, 0\right) \in \mathbb{R}^{n}$, we have

$$
\begin{aligned}
\Gamma(z)= & \left\{(\tau, \tau, \tau) \mid \tau \in \partial_{B} f\left(z_{1}\right)\right\} \\
& \cup\left\{\left(f_{-}^{\prime}\left(z_{1}\right), f_{+}^{\prime}\left(z_{1}\right), \partial f\left(z_{1}\right)\right)\right\} .
\end{aligned}
$$

Proof. It follows from the definition of $\Gamma(z)$ via (91) that

$$
\begin{aligned}
\Gamma(z)= & \lim _{\substack{t_{k}, t_{k}^{\prime} \rightarrow z_{1} \\
t_{k} t_{k}^{\prime} \in D_{f}, t_{k}<t_{k}^{\prime}}}\left(f^{\prime}\left(t_{k}\right), f^{\prime}\left(t_{k}^{\prime}\right), \frac{f\left(t_{k}^{\prime}\right)-f\left(t_{k}\right)}{t_{k}^{\prime}-t_{k}}\right) \\
= & \limsup _{\substack{t_{k}, t_{k}^{\prime} \rightarrow z_{1} \\
t_{k}, t_{k}^{\prime} \in D_{f}, t_{k}<t_{k}^{\prime} \leq z_{1}}}\left(f^{\prime}\left(t_{k}\right), f^{\prime}\left(t_{k}^{\prime}\right), \frac{f\left(t_{k}^{\prime}\right)-f\left(t_{k}\right)}{t_{k}^{\prime}-t_{k}}\right) \\
& +\limsup _{\substack{t_{k}, t_{k}^{\prime} \rightarrow z_{1} \\
t_{k} t_{k}^{\prime} \in D_{f}, z_{1} \leq t_{k}<t_{k}^{\prime}}}\left(f^{\prime}\left(t_{k}\right), f^{\prime}\left(t_{k}^{\prime}\right), \frac{f\left(t_{k}^{\prime}\right)-f\left(t_{k}\right)}{t_{k}^{\prime}-t_{k}}\right) \\
& +\limsup _{\substack{t_{k}, t_{k}^{\prime} \rightarrow z_{1} \\
t_{k}, t_{k}^{\prime} \in D_{f}, t_{k}<z_{1}<t_{k}^{\prime}}}\left(f^{\prime}\left(t_{k}\right), f^{\prime}\left(t_{k}^{\prime}\right), \frac{f\left(t_{k}^{\prime}\right)-f\left(t_{k}\right)}{t_{k}^{\prime}-t_{k}}\right) .
\end{aligned}
$$

According to (104), it is easy to see that

$$
\begin{gathered}
\partial_{B} f\left(z_{1}\right)=\left\{f_{-}^{\prime}\left(z_{1}\right), f_{+}^{\prime}\left(z_{1}\right)\right\}, \\
\partial f\left(z_{1}\right)=\left[f_{-}^{\prime}\left(z_{1}\right), f_{+}^{\prime}\left(z_{1}\right)\right],
\end{gathered}
$$

$$
\begin{aligned}
& \limsup _{\substack{t_{k}, t_{k}^{\prime} \rightarrow z_{1} \\
t_{k}, t_{k}^{\prime} \in D_{f}, t_{k}<t_{k}^{\prime} \leq z_{1}}}\left(f^{\prime}\left(t_{k}\right), f^{\prime}\left(t_{k}^{\prime}\right), \frac{f\left(t_{k}^{\prime}\right)-f\left(t_{k}\right)}{t_{k}^{\prime}-t_{k}}\right) \\
& =\left(f_{-}^{\prime}\left(z_{1}\right), f_{-}^{\prime}\left(z_{1}\right), f_{-}^{\prime}\left(z_{1}\right)\right),
\end{aligned}
$$

$$
\begin{aligned}
& \limsup _{\substack{t_{k}, t_{k}^{\prime} \rightarrow z_{1} \\
t_{k}, t_{k}^{\prime} \in D_{f}, z_{1} \leq t_{k}<t_{k}^{\prime}}}\left(f^{\prime}\left(t_{k}\right), f^{\prime}\left(t_{k}^{\prime}\right), \frac{f\left(t_{k}^{\prime}\right)-f\left(t_{k}\right)}{t_{k}^{\prime}-t_{k}}\right) \\
& =\left(f_{+}^{\prime}\left(z_{1}\right), f_{+}^{\prime}\left(z_{1}\right), f_{+}^{\prime}\left(z_{1}\right)\right) .
\end{aligned}
$$

These are the elements of $(\tau, \tau, \tau)$ with $\tau \in \partial_{B} f\left(z_{1}\right)$, since $\partial_{B} f\left(z_{1}\right)=\left\{f_{-}^{\prime}\left(z_{1}\right), f_{+}^{\prime}\left(z_{1}\right)\right\}$. Now we claim that

$$
\begin{aligned}
& \lim _{\substack{t_{k}, t_{k}^{\prime} \rightarrow z_{1} \\
t_{k}, t_{k}^{\prime} \in D_{f}, t_{k}<z_{1}<t_{k}^{\prime}}}\left(f^{\prime}\left(t_{k}\right), f^{\prime}\left(t_{k}^{\prime}\right), \frac{f\left(t_{k}^{\prime}\right)-f\left(t_{k}\right)}{t_{k}^{\prime}-t_{k}}\right) \\
& \quad=\left(f_{-}^{\prime}\left(z_{1}\right), f_{+}^{\prime}\left(z_{1}\right), \partial f\left(z_{1}\right)\right) .
\end{aligned}
$$

It only needs to show

$$
\limsup _{\substack{t_{k}, t_{k}^{\prime} \rightarrow z_{1} \\ s_{k} \in D_{f}, t_{k}<z_{1}<t_{k}^{\prime}}} \frac{f\left(t_{k}^{\prime}\right)-f\left(t_{k}\right)}{t_{k}^{\prime}-t_{k}}=\partial f\left(z_{1}\right) .
$$


First, we observe that

$$
\begin{aligned}
& \frac{f\left(t_{k}^{\prime}\right)-f\left(t_{k}\right)}{t_{k}^{\prime}-t_{k}} \\
& \quad=\frac{t_{k}^{\prime}-z_{1}}{t_{k}^{\prime}-t_{k}} \frac{f\left(t_{k}^{\prime}\right)-f\left(z_{1}\right)}{t_{k}^{\prime}-z_{1}}+\frac{z_{1}-t_{k}}{t_{k}^{\prime}-t_{k}} \frac{f\left(z_{1}\right)-f\left(t_{k}\right)}{z_{1}-t_{k}} \\
& \longrightarrow(1-r) f_{+}^{\prime}\left(z_{1}\right)+r f_{-}^{\prime}\left(z_{1}\right) \in \partial f\left(z_{1}\right),
\end{aligned}
$$

for some $r \in[0,1]$. Conversely, taking $\tau \in \partial f\left(z_{1}\right)$ and using $\partial f\left(z_{1}\right)=\left[f_{-}^{\prime}\left(z_{1}\right), f_{+}^{\prime}\left(z_{1}\right)\right]$ yield $\tau=(1-r) f_{+}^{\prime}\left(z_{1}\right)+r f_{-}^{\prime}\left(z_{1}\right)$ for some $r \in[0,1]$. Due to $D_{f}$ being dense in $\mathbb{R}$, for any $k \geq 1$, we define

$$
\begin{aligned}
& T_{1}:=D_{f} \cap\left[z_{1}-r \frac{1}{k}-\frac{1}{k^{2}}, z_{1}-r \frac{1}{k}\right] \neq \emptyset \\
& T_{2}:=D_{f} \cap\left[z_{1}+(1-r) \frac{1}{k}, z_{1}+(1-r) \frac{1}{k}+\frac{1}{k^{2}}\right] \neq \emptyset .
\end{aligned}
$$

Take $t_{k} \in T_{1}$ and $t_{k}^{\prime} \in T_{2}$; then for $t_{k}^{\prime}>z_{1}>t_{k}$, we have

$$
\begin{aligned}
(1-r) \frac{1}{k} & \leq t_{k}^{\prime}-z_{1} \leq(1-r) \frac{1}{k}+\frac{1}{k^{2}}, \\
\frac{1}{k} & \leq t_{k}^{\prime}-t_{k} \leq \frac{1}{k}+\frac{2}{k^{2}},
\end{aligned}
$$

which imply

$$
\frac{(1-r)(1 / k)}{(1 / k)+\left(2 / k^{2}\right)} \leq \frac{t_{k}^{\prime}-z_{1}}{t_{k}^{\prime}-t_{k}} \leq \frac{(1-r)(1 / k)+\left(1 / k^{2}\right)}{1 / k} .
$$

Thus,

$$
\frac{t_{k}^{\prime}-z_{1}}{t_{k}^{\prime}-t_{k}} \longrightarrow 1-r
$$

Similarly, we have

$$
\frac{z_{1}-t_{k}}{t_{k}^{\prime}-t_{k}} \longrightarrow r
$$

In summary,

$$
\begin{aligned}
\tau & =(1-r) f_{+}^{\prime}\left(z_{1}\right)+r f_{-}^{\prime}\left(z_{1}\right) \\
& =\lim _{k \rightarrow \infty} \frac{t_{k}^{\prime}-z_{1}}{t_{k}^{\prime}-t_{k}} \frac{f\left(t_{k}^{\prime}\right)-f\left(z_{1}\right)}{t_{k}^{\prime}-z_{1}}+\frac{z_{1}-t_{k}}{t_{k}^{\prime}-t_{k}} \frac{f\left(z_{1}\right)-f\left(t_{k}\right)}{z_{1}-t_{k}} \\
& =\lim _{k \rightarrow \infty} \frac{f\left(t_{k}^{\prime}\right)-f\left(t_{k}\right)}{t_{k}^{\prime}-t_{k}} .
\end{aligned}
$$

This completes the proof.
Corollary 16. Suppose $f: \mathbb{R} \rightarrow \mathbb{R}$ is strictly continuous and directionally differentiable function satisfying

$$
\begin{aligned}
\lim _{\substack{t, v \rightarrow \tau^{\sigma} \\
t \neq v}} \frac{f(t)-f(v)}{t-v} & =\lim _{\substack{t \rightarrow \tau^{\sigma} \\
t \in D_{f}}} f^{\prime}(t)=f_{\sigma}^{\prime}(\tau), \\
& \forall \tau \in \mathbb{R}, \quad \sigma \in\{-,+\} .
\end{aligned}
$$

Then, for any $z=\left(z_{1}, 0\right) \in \mathbb{R}^{n}$,

$$
\begin{aligned}
\partial_{B}\left(f^{\mathscr{L}_{\theta}}\right)(z)=\left\{\left[\begin{array}{cc}
\xi & \varrho w^{T} \\
\varrho w & a I+(\eta-a) w w^{T}
\end{array}\right] \mid\right. \\
\quad \text { either } a=\xi \in \partial_{B} f\left(z_{1}\right), \varrho=0, \\
\quad \text { or } a \in \partial f\left(z_{1}\right), \\
\xi-\varrho \operatorname{ctan} \theta=f_{-}^{\prime}\left(z_{1}\right), \\
\xi+\varrho \tan \theta=f_{+}^{\prime}\left(z_{1}\right), \\
\eta=\xi-\varrho(\operatorname{ctan} \theta-\tan \theta), \\
\|w\|=1\} .
\end{aligned}
$$

Proof. This result follows by combining Theorem 13 and Lemma 15.

We point out that if $\theta=45^{\circ}$, then Corollary 16 reduces to [5, Lemma 5].

\section{Directional Differentiability in Hadamard Sense, Positive Homogeneity, and Boundedness}

In this section, we study some other important properties between $f^{\mathscr{L}_{\theta}}$ and $f$, such as directional differentiability in the Hadamard sense, positive homogeneity, and boundedness. These are new discoveries and are not studied in $[1,5,6]$ or other settings. First, we note that if a function is directionally differentiable in the Hadamard senses, then it must be directionally differentiable. The converse statement holds true if this function is from $\mathbb{R}$ to $\mathbb{R}$, since in this case the direction just has \pm 1 . More precisely, for a real-valued function $g$ : $\mathbb{R} \rightarrow \mathbb{R}, g$ is directionally differentiable if and only if $g$ is directionally differentiable in the Hadamard sense. Indeed, in the proof of [5, Proposition 3], the authors already employ the property of directionally differentiable in the Hadamard sense and even the assumption is directionally differentiable. However, for general mappings $g: \mathbb{R}^{n}$ to $\mathbb{R}$ or $g: \mathbb{R}^{n} \rightarrow \mathbb{R}^{m}$, these two concepts are not equivalent. In other words, if $g$ is directionally differentiable in the Hadamard sense, then $g$ is directionally differentiable. But, the converse is invalid in general, unless some additional assumption is imposed; for example, local Lipschitz continuity [20]. Nonetheless, we will show for the special function $f^{\mathscr{L}_{\theta}}: \mathbb{R}^{n} \rightarrow \mathbb{R}^{n}$, these two concepts are still equivalent. 
Theorem 17. Let $f: \mathbb{R} \rightarrow \mathbb{R}$ and $f^{\mathscr{L}_{\theta}}$ be defined as in (4). Then, $f^{\mathscr{L}_{\theta}}$ is directionally differentiable at $z$ if and only if $f^{\mathscr{L}_{\theta}}$ is directionally differentiable at $z$ in the Hadamard sense.

Proof. " $\models$ " This direction is clear.

“ $\Rightarrow$ " Suppose that $f^{\mathscr{L}_{\theta}}$ is directionally differentiable at $z$. Then, from Lemma $1, f$ is directionally differentiable, and hence $f$ is directionally differentiable in the Hadamard sense, since $f$ is a function from $\mathbb{R}$ to $\mathbb{R}$. To proceed the proof, we consider the following three cases.

Case 1. $z_{2}=0$ and $h_{2}=0$. Let $h^{\prime}=\left(h_{1}^{\prime}, h_{2}^{\prime}\right) \rightarrow h=\left(h_{1}, h_{2}\right)$. If $h_{2}^{\prime}=0$, then the proof is trivial. If $h_{2}^{\prime} \neq 0$, then

$$
\begin{aligned}
& f^{\mathscr{L}_{\theta}}\left(z+t h^{\prime}\right)-f^{\mathscr{L}_{\theta}}(z) \\
& =\left[\begin{array}{c}
\frac{f\left(z_{1}+t h_{1}^{\prime}-t\left\|h_{2}^{\prime}\right\| \operatorname{ctan} \theta\right)}{1+\operatorname{ctan}^{2} \theta}+\frac{f\left(z_{1}+t h_{1}^{\prime}+t\left\|h_{2}^{\prime}\right\| \tan \theta\right)}{1+\tan ^{2} \theta} \\
\left(-\frac{f\left(z_{1}+t h_{1}^{\prime}-t\left\|h_{2}^{\prime}\right\| \operatorname{ctan} \theta\right) \operatorname{ctan} \theta}{1+\operatorname{ctan}^{2} \theta}+\frac{f\left(z_{1}+t h_{1}^{\prime}+t\left\|h_{2}^{\prime}\right\| \tan \theta\right) \tan \theta}{1+\tan ^{2} \theta}\right) \frac{h_{2}^{\prime}}{\left\|h_{2}^{\prime}\right\|}
\end{array}\right] \\
& -\left[\begin{array}{c}
\frac{f\left(z_{1}\right)}{1+\operatorname{ctan}^{2} \theta}+\frac{f\left(z_{1}\right)}{1+\tan ^{2} \theta} \\
\left(-\frac{f\left(z_{1}\right) \operatorname{ctan} \theta}{1+\operatorname{ctan}^{2} \theta}+\frac{f\left(z_{1}\right) \tan \theta}{1+\tan ^{2} \theta}\right) w
\end{array}\right],
\end{aligned}
$$

where $w$ is taken to be $h_{2}^{\prime} /\left\|h_{2}^{\prime}\right\|$. Since $f$ is directionally differentiable in the Hadamard sense, then

$$
\begin{aligned}
& \lim _{\substack{h^{\prime} \rightarrow h \\
t \downarrow 0}} \frac{f\left(z_{1}+t h_{1}^{\prime}-t\left\|h_{2}^{\prime}\right\| \operatorname{ctan} \theta\right)-f\left(z_{1}\right)}{t}=f^{\prime}\left(z_{1} ; h_{1}\right), \\
& \lim _{\substack{h^{\prime} \rightarrow h \\
t \downarrow 0}} \frac{f\left(z_{1}+t h_{1}^{\prime}-t\left\|h_{2}^{\prime}\right\| \tan \theta\right)-f\left(z_{1}\right)}{t}=f^{\prime}\left(z_{1} ; h_{1}\right) .
\end{aligned}
$$

Therefore,

$$
\begin{gathered}
\frac{1}{t}\left[\frac{f\left(z_{1}+t h_{1}^{\prime}-t\left\|h_{2}^{\prime}\right\| \operatorname{ctan} \theta\right)}{1+\operatorname{ctan}^{2} \theta}+\frac{f\left(z_{1}+t h_{1}^{\prime}+t\left\|h_{2}^{\prime}\right\| \tan \theta\right)}{1+\tan ^{2} \theta}\right. \\
\left.-\frac{f\left(z_{1}\right)}{1+\operatorname{ctan}^{2} \theta}-\frac{f\left(z_{1}\right)}{1+\tan ^{2} \theta}\right] \\
\quad \longrightarrow \frac{f^{\prime}\left(z_{1} ; h_{1}\right)}{1+\operatorname{ctan}^{2} \theta}+\frac{f^{\prime}\left(z_{1} ; h_{1}\right)}{1+\tan ^{2} \theta}=f^{\prime}\left(z_{1} ; h_{1}\right),
\end{gathered}
$$

$$
\begin{gathered}
\frac{1}{t}\left\{\left[-\frac{f\left(z_{1}+t h_{1}^{\prime}-t\left\|h_{2}^{\prime}\right\| \operatorname{ctan} \theta\right) \operatorname{ctan} \theta}{1+\operatorname{ctan}^{2} \theta}\right.\right. \\
\left.+\frac{f\left(z_{1}+t h_{1}^{\prime}+t\left\|h_{2}^{\prime}\right\| \tan \theta\right) \tan \theta}{1+\tan ^{2} \theta}\right] \\
\left.-\left[-\frac{f\left(z_{1}\right) \operatorname{ctan} \theta}{1+\operatorname{ctan}^{2} \theta}+\frac{f\left(z_{1}\right) \tan \theta}{1+\tan ^{2} \theta}\right]\right\} \frac{h_{2}^{\prime}}{\left\|h_{2}^{\prime}\right\|} \longrightarrow 0
\end{gathered}
$$

because the term in big brace approaches to zero and $h_{2}^{\prime} /\left\|h_{2}^{\prime}\right\|$ is bounded. These two limits imply

$$
\begin{aligned}
\lim _{\substack{h^{\prime} \rightarrow h \\
t \downarrow 0}} \frac{f^{\mathscr{L}_{\theta}}\left(z+t h^{\prime}\right)-f^{\mathscr{L}_{\theta}}(z)}{t} & =\left[\begin{array}{c}
f^{\prime}\left(z_{1} ; h_{1}\right) \\
0
\end{array}\right]=f^{\prime}\left(z_{1} ; h_{1}\right) e \\
& =\left(f^{\mathscr{L}_{\theta}}\right)^{\prime}(z ; h),
\end{aligned}
$$

which says $f^{\mathscr{L}_{\theta}}$ is directionally differentiable at $z$ in the Hadamard sense. 
Case 2. $z_{2}=0$ and $h_{2} \neq 0$. Note that

$$
\begin{aligned}
& f^{\mathscr{L}_{\theta}}\left(z+t h^{\prime}\right)-f^{\mathscr{L}_{\theta}}(z) \\
& =\left[\begin{array}{c}
\frac{f\left(z_{1}+t h_{1}^{\prime}-t\left\|h_{2}^{\prime}\right\| \operatorname{ctan} \theta\right)}{1+\operatorname{ctan}^{2} \theta}+\frac{f\left(z_{1}+t h_{1}^{\prime}+t\left\|h_{2}^{\prime}\right\| \tan \theta\right)}{1+\tan ^{2} \theta} \\
\left(-\frac{f\left(z_{1}+t h_{1}^{\prime}-t\left\|h_{2}^{\prime}\right\| \operatorname{ctan} \theta\right) \operatorname{ctan} \theta}{1+\operatorname{ctan}^{2} \theta}+\frac{f\left(z_{1}+t h_{1}^{\prime}+t\left\|h_{2}^{\prime}\right\| \tan \theta\right) \tan \theta}{1+\tan ^{2} \theta}\right) \frac{h_{2}^{\prime}}{\left\|h_{2}^{\prime}\right\|}
\end{array}\right]-\left[\begin{array}{c}
f\left(z_{1}\right) \\
0
\end{array}\right] \\
& =\left[\begin{array}{c}
\frac{f\left(z_{1}+t h_{1}^{\prime}-t\left\|h_{2}^{\prime}\right\| \operatorname{ctan} \theta\right)}{1+\operatorname{ctan}^{2} \theta}+\frac{f\left(z_{1}+t h_{1}^{\prime}+t\left\|h_{2}^{\prime}\right\| \tan \theta\right)}{1+\tan ^{2} \theta}-f\left(z_{1}\right) \\
\left(-\frac{f\left(z_{1}+t h_{1}^{\prime}-t\left\|h_{2}^{\prime}\right\| \operatorname{ctan} \theta\right) \operatorname{ctan} \theta}{1+\operatorname{ctan}^{2} \theta}+\frac{f\left(z_{1}+t h_{1}^{\prime}+t\left\|h_{2}^{\prime}\right\| \tan \theta\right) \tan \theta}{1+\tan ^{2} \theta}\right) \frac{h_{2}^{\prime}}{\left\|h_{2}^{\prime}\right\|}
\end{array}\right] .
\end{aligned}
$$

For the first component, we compute that

$$
\begin{gathered}
\frac{1}{t}\left[\frac{f\left(z_{1}+t h_{1}^{\prime}-t\left\|h_{2}^{\prime}\right\| \operatorname{ctan} \theta\right)}{1+\operatorname{ctan}^{2} \theta}+\frac{f\left(z_{1}+t h_{1}^{\prime}+t\left\|h_{2}^{\prime}\right\| \tan \theta\right)}{1+\tan ^{2} \theta}\right. \\
\left.-f\left(z_{1}\right)\right] \\
=\frac{1}{t}\left[\frac{f\left(z_{1}+t\left(h_{1}^{\prime}-\left\|h_{2}^{\prime}\right\| \operatorname{ctan} \theta\right)\right)-f\left(z_{1}\right)}{1+\operatorname{ctan}^{2} \theta}\right. \\
\left.+\frac{f\left(z_{1}+t\left(h_{1}^{\prime}+\left\|h_{2}^{\prime}\right\| \tan \theta\right)\right)-f\left(z_{1}\right)}{1+\tan ^{2} \theta}\right] \\
\longrightarrow \frac{f^{\prime}\left(z_{1} ; h_{1}-\left\|h_{2}\right\| \operatorname{ctan} \theta\right)}{1+\operatorname{ctan}^{2} \theta}+\frac{f^{\prime}\left(z_{1} ; h_{1}+\left\|h_{2}\right\| \tan \theta\right)}{1+\tan ^{2} \theta} \\
\text { as } t \downarrow 0, h^{\prime} \rightarrow h,
\end{gathered}
$$

For the second component, we have

$$
\begin{gathered}
\frac{1}{t}\left[-\frac{f\left(z_{1}+t h_{1}^{\prime}-t\left\|h_{2}^{\prime}\right\| \operatorname{ctan} \theta\right) \operatorname{ctan} \theta}{1+\operatorname{ctan}^{2} \theta}\right. \\
\left.+\frac{f\left(z_{1}+t h_{1}^{\prime}+t\left\|h_{2}^{\prime}\right\| \tan \theta\right) \tan \theta}{1+\tan ^{2} \theta}\right] \frac{h_{2}^{\prime}}{\left\|h_{2}^{\prime}\right\|} \\
\longrightarrow\left[-\frac{\operatorname{ctan} \theta}{1+\operatorname{ctan}^{2} \theta} f^{\prime}\left(z_{1} ; h_{1}-\left\|h_{2}\right\| \operatorname{ctan} \theta\right)\right. \\
\left.\quad+\frac{\tan \theta}{1+\tan ^{2} \theta} f^{\prime}\left(z_{1} ; h_{1}+\left\|h_{2}\right\| \tan \theta\right)\right] \frac{h_{2}}{\left\|h_{2}\right\|} .
\end{gathered}
$$

The above two limits show that

$$
\begin{aligned}
& \lim _{\substack{h^{\prime} \rightarrow 0 \\
t \downarrow 0}} \frac{f^{\mathscr{L}_{\theta}}\left(z+t h^{\prime}\right)-f^{\mathscr{L}_{\theta}}(z)}{t}=\frac{1}{1+\operatorname{ctan}^{2} \theta} \\
& \quad \times f^{\prime}\left(z_{1} ; h_{1}-\left\|h_{2}\right\| \operatorname{ctan} \theta\right)\left[\begin{array}{cc}
1 & 0 \\
0 & \operatorname{ctan} \theta \cdot I
\end{array}\right]\left[\begin{array}{c}
1 \\
-\frac{h_{2}}{\left\|h_{2}\right\|}
\end{array}\right]
\end{aligned}
$$

$$
+\frac{1}{1+\tan ^{2} \theta} f^{\prime}\left(z_{1} ; h_{1}+\left\|h_{2}\right\| \tan \theta\right)\left[\begin{array}{cc}
1 & 0 \\
0 & \tan \theta \cdot I
\end{array}\right]\left[\begin{array}{c}
1 \\
\frac{h_{2}}{\left\|h_{2}\right\|}
\end{array}\right]
$$$$
=\left(f^{\mathscr{L}_{\theta}}\right)^{\prime}(z ; h) .
$$
differentiable in the Hadamard sense. 
Case $3 . z_{2} \neq 0$. Then

$$
\begin{aligned}
& f^{\mathscr{L}_{\theta}}\left(z+t h^{\prime}\right)-f^{\mathscr{L}_{\theta}}(z) \\
& =\left[\begin{array}{c}
\frac{f\left(z_{1}+t h_{1}^{\prime}-\left\|z_{2}+t h_{2}^{\prime}\right\| \operatorname{ctan} \theta\right)}{1+\operatorname{ctan}^{2} \theta}+\frac{f\left(z_{1}+t h_{1}^{\prime}+\left\|z_{2}+t h_{2}^{\prime}\right\| \tan \theta\right)}{1+\tan ^{2} \theta} \\
\left(-\frac{f\left(z_{1}+t h_{1}^{\prime}-\left\|z_{2}+t h_{2}^{\prime}\right\| \operatorname{ctan} \theta\right) \operatorname{ctan} \theta}{1+\operatorname{ctan}^{2} \theta}+\frac{f\left(z_{1}+t h_{1}^{\prime}+\left\|z_{2}+t h_{2}^{\prime}\right\| \tan \theta\right) \tan \theta}{1+\tan ^{2} \theta}\right) \frac{z_{2}+t h_{2}^{\prime}}{\left\|z_{2}+t h_{2}^{\prime}\right\|}
\end{array}\right] \\
& -\left[\begin{array}{c}
\frac{f\left(z_{1}-\left\|z_{2}\right\| \operatorname{ctan} \theta\right)}{1+\operatorname{ctan}^{2} \theta}+\frac{f\left(z_{1}+\left\|z_{2}\right\| \tan \theta\right)}{1+\tan ^{2} \theta} \\
\left(-\frac{f\left(z_{1}-\left\|z_{2}\right\| \operatorname{ctan} \theta\right) \operatorname{ctan} \theta}{1+\operatorname{ctan}^{2} \theta}+\frac{f\left(z_{1}+\left\|z_{2}\right\| \tan \theta\right) \tan \theta}{1+\tan ^{2} \theta}\right) \frac{z_{2}}{\left\|z_{2}\right\|}
\end{array}\right] .
\end{aligned}
$$

Then, the first component of $\left(f^{\mathscr{L}_{\theta}}\left(z+t h^{\prime}\right)-f^{\mathscr{L}_{\theta}}(z)\right) / t$ behaves as follows (when $t \downarrow 0$ and $h^{\prime} \rightarrow h$ ):

$$
\begin{aligned}
& \frac{1}{t}\left[\frac{f\left(z_{1}+t h_{1}^{\prime}-\left\|z_{2}+t h_{2}^{\prime}\right\| \operatorname{ctan} \theta\right)}{1+\operatorname{ctan}^{2} \theta}\right. \\
& +\frac{f\left(z_{1}+t h_{1}^{\prime}+\left\|z_{2}+t h_{2}^{\prime}\right\| \tan \theta\right)}{1+\tan ^{2} \theta} \\
& \left.-\frac{f\left(z_{1}-\left\|z_{2}\right\| \operatorname{ctan} \theta\right)}{1+\operatorname{ctan}^{2} \theta}-\frac{f\left(z_{1}+\left\|z_{2}\right\| \tan \theta\right)}{1+\tan ^{2} \theta}\right] \\
& =\frac{1}{t}\left[\frac{f\left(z_{1}+t h_{1}^{\prime}-\left\|z_{2}+t h_{2}^{\prime}\right\| \operatorname{ctan} \theta\right)-f\left(z_{1}-\left\|z_{2}\right\| \operatorname{ctan} \theta\right)}{1+\operatorname{ctan}^{2} \theta}\right. \\
& \left.+\frac{f\left(z_{1}+t h_{1}^{\prime}+\left\|z_{2}+t h_{2}^{\prime}\right\| \tan \theta\right)-f\left(z_{1}+\left\|z_{2}\right\| \tan \theta\right)}{1+\tan ^{2} \theta}\right] \\
& =\frac{1}{t}\left[\left(f\left(z_{1}+t h_{1}^{\prime}-\left\|z_{2}\right\| \operatorname{ctan} \theta-t \frac{z_{2}^{T} h_{2}^{\prime}}{\left\|z_{2}\right\|} \operatorname{ctan} \theta+o(t)\right)\right.\right. \\
& \left.-f\left(z_{1}-\left\|z_{2}\right\| \operatorname{ctan} \theta\right)\right) \\
& \times\left(1+\operatorname{ctan}^{2} \theta\right)^{-1} \\
& +\left(f\left(z_{1}+t h_{1}^{\prime}+\left\|z_{2}\right\| \tan \theta+t \frac{z_{2}^{T} h_{2}^{\prime}}{\left\|z_{2}\right\|} \tan \theta+o(t)\right)\right. \\
& \left.-f\left(z_{1}+\left\|z_{2}\right\| \tan \theta\right)\right) \\
& \left.\times\left(1+\tan ^{2} \theta\right)^{-1}\right]
\end{aligned}
$$

$$
\begin{aligned}
& \rightarrow \frac{f^{\prime}\left(z_{1}-\left\|z_{2}\right\| \operatorname{ctan} \theta ; h_{1}-\left(z_{2}^{T} h_{2} /\left\|z_{2}\right\|\right) \operatorname{ctan} \theta\right)}{1+\operatorname{ctan}^{2} \theta} \\
&+\frac{f^{\prime}\left(z_{1}+\left\|z_{2}\right\| \tan \theta ; h_{1}+\left(z_{2}^{T} h_{2} /\left\|z_{2}\right\|\right) \tan \theta\right)}{1+\tan ^{2} \theta}
\end{aligned}
$$

where in the last step we have used the fact that $f$ is directionally differentiable in the Hadamard sense. Recall that $\Phi\left(z_{2}\right)=z_{2} /\left\|z_{2}\right\|$ is continuously differentiable at $z_{2} \neq 0$. Then, the second component of $\left(f^{\mathscr{L}_{\theta}}\left(z+t h^{\prime}\right)-f^{\mathscr{L}_{\theta}}(z)\right) / t$ behaves as follows (when $t \downarrow 0$ and $h^{\prime} \rightarrow h$ ):

$$
\begin{aligned}
& \frac{1}{t}\left\{\left[-\frac{f\left(z_{1}+t h_{1}^{\prime}-\left\|z_{2}+t h_{2}^{\prime}\right\| \operatorname{ctan} \theta\right) \operatorname{ctan} \theta}{1+\operatorname{ctan}^{2} \theta}\right.\right. \\
& \left.+\frac{f\left(z_{1}+t h_{1}^{\prime}+\left\|z_{2}+t h_{2}^{\prime}\right\| \tan \theta\right) \tan \theta}{1+\tan ^{2} \theta}\right] \frac{z_{2}+t h_{2}^{\prime}}{\left\|z_{2}+t h_{2}^{\prime}\right\|} \\
& -\left[-\frac{f\left(z_{1}-\left\|z_{2}\right\| \operatorname{ctan} \theta\right) \operatorname{ctan} \theta}{1+\operatorname{ctan}^{2} \theta}\right. \\
& \left.\left.\longrightarrow+\frac{f\left(z_{1}+\left\|z_{2}\right\| \tan \theta\right) \tan \theta}{1+\tan ^{2} \theta}\right] \frac{z_{2}}{\left\|z_{2}\right\|}\right\} \\
& \quad\left[-\frac{\operatorname{ctan}^{2}}{1+\operatorname{ctan}^{2} \theta} f^{\prime}\left(z_{1}-\left\|z_{2}\right\| \operatorname{ctan} \theta ; h_{1}-\frac{z_{2}^{T} h_{2}}{\left\|z_{2}\right\|} \operatorname{ctan} \theta\right)\right. \\
& \left.+\frac{\tan \theta}{1+\tan ^{2} \theta} f^{\prime}\left(z_{1}+\left\|z_{2}\right\| \tan \theta ; h_{1}+\frac{z_{2}^{T} h_{2}}{\left\|z_{2}\right\|} \tan \theta\right)\right]
\end{aligned}
$$




$$
\begin{array}{r}
\times \frac{z_{2}}{\left\|z_{2}\right\|}+\left[-\frac{f\left(z_{1}-\left\|z_{2}\right\| \operatorname{ctan} \theta\right) \operatorname{ctan} \theta}{1+\operatorname{ctan}^{2} \theta}\right. \\
\left.+\frac{f\left(z_{1}+\left\|z_{2}\right\| \tan \theta\right) \tan \theta}{1+\tan ^{2} \theta}\right] \\
\times\left[\frac{1}{\left\|z_{2}\right\|}\left(I-\frac{z_{2} z_{2}^{T}}{\left\|z_{2}\right\|^{2}}\right)\right] h_{2} .
\end{array}
$$

The above two limits show that

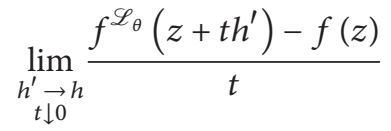

$$
\begin{aligned}
& =\frac{1}{1+\operatorname{ctan}^{2} \theta} f^{\prime}\left(\lambda_{1}(z) ; h_{1}-\frac{z_{2}^{T} h_{2}}{\left\|z_{2}\right\|} \operatorname{ctan} \theta\right) \\
& \times\left[\begin{array}{cc}
1 & 0 \\
0 & \operatorname{ctan} \theta \cdot I
\end{array}\right]\left[\begin{array}{c}
1 \\
-\frac{z_{2}}{\left\|z_{2}\right\|}
\end{array}\right] \\
& -\frac{\operatorname{ctan} \theta}{1+\operatorname{ctan}^{2} \theta} \frac{f\left(\lambda_{1}(z)\right)}{\left\|z_{2}\right\|} M_{z_{2}} h \\
& +\frac{1}{1+\tan ^{2} \theta} f^{\prime}\left(\lambda_{2}(z) ; h_{1}+\frac{z_{2}^{T} h_{2}}{\left\|z_{2}\right\|} \tan \theta\right) \\
& \times\left[\begin{array}{cc}
1 & 0 \\
0 & \tan \theta \cdot I
\end{array}\right]\left[\begin{array}{c}
1 \\
\frac{z_{2}}{\left\|z_{2}\right\|}
\end{array}\right] \\
& +\frac{\tan \theta}{1+\tan ^{2} \theta} \frac{f\left(\lambda_{2}(z)\right)}{\left\|z_{2}\right\|} M_{z_{2}} h=\left(f^{\mathscr{L}_{\theta}}\right)^{\prime}(z ; h) \text {. }
\end{aligned}
$$

The proof is complete.

Theorem 18. Let $f: \mathbb{R} \rightarrow \mathbb{R}$ and $f^{\mathscr{L}_{\theta}}$ be defined as in (4). Then, the following statements are equivalent.

(a) $f^{\mathscr{L}_{\theta}}$ is directionally differentiable at $z$;

(b) $f^{\mathscr{L}_{\theta}}$ is directionally differentiable at $z$ in the Hadamard sense;

(c) $f$ is directionally differentiable at $\lambda_{i}(z)$, for $i=1,2$;

(d) $f$ is directionally differentiable at $\lambda_{i}(z)$, for $i=1,2$ in the Hadamard sense.

Proof. The equivalence $(a) \Leftrightarrow(b)$ comes from Theorem 17; $(c) \Leftrightarrow(d)$ is due to the fact that $f: \mathbb{R} \rightarrow \mathbb{R} ;(a) \Leftrightarrow(c)$ follows from Lemma 1.

Below we study the relationship of positive homogeneity and boundedness between $f^{\mathscr{L}_{\theta}}$ and $f$.

Theorem 19. Let $f: \mathbb{R} \rightarrow \mathbb{R}$ and $f^{\mathscr{L}_{\theta}}$ be defined as in (4). Then, $f^{\mathscr{L}_{\theta}}$ is positively homogeneous at $z$ with exponent $\alpha>0$ if and only if $f$ is positively homogeneous at $\lambda_{i}(z)$ for $i=1,2$ with exponent $\alpha>0$.
Proof. " $\Leftarrow$ " Suppose that $f$ is positively homogeneous at $\lambda_{i}(z)$ for $i=1,2$ with exponent $\alpha>0$. For any $z=\left(z_{1}, z_{2}\right) \in$ $\mathbb{R} \times \mathbb{R}^{n-1}$ with $z=\lambda_{1}(z) u_{z}^{1}+\lambda_{2}(z) u_{z}^{2}$, we observe that $u_{k z}^{i}=u_{z}^{i}$, $i=1,2$ for $k \geq 0$, whenever $z_{2} \neq 0$ and $\lambda_{i}(k z)=k \lambda_{i}(z)$, for $i=1,2$. Hence, when $z_{2} \neq 0$, we have

$$
\begin{aligned}
f^{\mathscr{L}_{\theta}}(k z) & =f\left(\lambda_{1}(k z)\right) u_{k z}^{1}+f\left(\lambda_{2}(k z)\right) u_{k z}^{2} \\
& =f\left(k \lambda_{1}(z)\right) u_{z}^{1}+f\left(k \lambda_{2}(z)\right) u_{z}^{2} \\
& =k^{\alpha}\left[f\left(\lambda_{1}(z)\right) u_{z}^{1}+f\left(\lambda_{2}(z)\right) u_{z}^{2}\right] \\
& =k^{\alpha} f(z) .
\end{aligned}
$$

When $z_{2}=0$, we know that $\lambda_{i}(z)=z_{1}$ for $i=1,2$. Thus,

$$
f^{\mathscr{L}_{\theta}}(k z)=\left[\begin{array}{c}
f\left(k z_{1}\right) \\
0
\end{array}\right]=\left[\begin{array}{c}
k^{\alpha} f\left(z_{1}\right) \\
0
\end{array}\right]=k^{\alpha} f^{\mathscr{L}_{\theta}}(z) .
$$

All the above shows that $f^{\mathscr{L}_{\theta}}$ is positively homogeneous at $z$ with exponent $\alpha>0$.

“ $\Rightarrow$ " Suppose that $f^{\mathscr{L}_{\theta}}$ is positively homogeneous at $z$ with exponent $\alpha>0$; that is, $f^{\mathscr{L}_{\theta}}(k z)=k^{\alpha} f^{\mathscr{L}_{\theta}}(z)$. Then, we have

$$
\begin{aligned}
f\left(k \lambda_{i}(z)\right)\left\|u_{z}^{i}\right\|^{2} & =\left\langle f^{\mathscr{L}_{\theta}}(k z), u_{z}^{i}\right\rangle=k^{\alpha}\left\langle f^{\mathscr{L}_{\theta}}(z), u_{z}^{i}\right\rangle \\
& =k^{\alpha} f\left(\lambda_{i}(z)\right)\left\|u_{z}^{i}\right\|^{2},
\end{aligned}
$$

which in turn implies $f\left(k \lambda_{i}(z)\right)=k^{\alpha} f\left(\lambda_{i}(z)\right)$, since $\left\|u_{z}^{i}\right\| \neq 0$. Hence, $f$ is positively homogenous at $\lambda_{i}(z)$ for $i=1,2$ with exponent $\alpha>0$.

Theorem 20. Let $f: \mathbb{R} \rightarrow \mathbb{R}$ and $f^{\mathscr{L}_{\theta}}$ be defined as in (4). Then, $f^{\mathscr{L}_{\theta}}$ is bounded if and only if $f$ is bounded.

Proof. " $\Leftarrow$ " Suppose that $f$ is bounded by $M$. The proof for this direction follows from the following inequality:

$$
\begin{aligned}
\left\|f^{\mathscr{L}_{\theta}}(z)\right\| & =\left\|f\left(\lambda_{1}(z)\right) u_{z}^{1}+f\left(\lambda_{2}(z)\right) u_{z}^{2}\right\| \\
& \leq\left|f\left(\lambda_{1}(z)\right)\right| \cdot\left\|u_{z}^{1}\right\|+\left|f\left(\lambda_{2}(z)\right)\right| \cdot\left\|u_{z}^{2}\right\| \\
& \leq\left(\frac{1}{\sqrt{1+\operatorname{ctan}^{2} \theta}}+\frac{1}{\sqrt{1+\tan ^{2} \theta}}\right) M .
\end{aligned}
$$

" $\Rightarrow$ " Suppose that $f^{\mathscr{L}_{\theta}}$ is bounded by $M$. This direction is trivial because for any $\tau \in \mathbb{R}$, one has $f^{\mathscr{L}_{\theta}}(\tau e)=(f(\tau), 0)^{T}$, and hence

$$
|f(\tau)|=\left\|\left(\begin{array}{c}
f(\tau) \\
0
\end{array}\right)\right\|=\left\|f^{\mathscr{L}_{\theta}}(\tau e)\right\| \leq M
$$

\section{Final Remarks}

So far, we have shown that many properties holding for $f^{\text {soc }}$ can be extended to the setting for $f^{\mathscr{L}_{\theta}}$. One may wonder 
whether $f^{\text {soc }}$ and $f^{\mathscr{L}_{\theta}}$ always share the same properties. The answer is no! Here, we present a simple property that holds for $f^{\text {soc }}$ but fails for $f^{\mathscr{L}_{\theta}}$. Some more different properties between $f^{\mathscr{L}_{\theta}}$ and $f^{\text {soc }}$ are discovered in [21].

To see the counterexample, we recall that a function $F$ : $\mathbb{R}^{n} \rightarrow \mathbb{R}^{n}$ is said to be an odd (even, resp.) function if $F(-x)=-F(x)(F(-x)=F(x)$, resp. $)$ for all $x \in \mathbb{R}^{n}$.

Proposition 21. Let $f: \mathbb{R} \rightarrow \mathbb{R}$ and $f^{s o c}$ be given as in (6). Then $f^{s o c}$ is an odd (even, resp.) function on $\mathbb{R}^{n}$ if and only if $f$ is an odd (even, resp.) function on $\mathbb{R}$.

Proof. “ $\Leftarrow$ ” In the setting of second-order cone, we observe that

$$
\begin{array}{cl}
u_{-z}^{1}=u_{z}^{2}, & u_{-z}^{2}=u_{z}^{1} \\
\lambda_{1}(-z)=-\lambda_{2}(z), & \lambda_{2}(-z)=-\lambda_{1}(z),
\end{array}
$$

which implies

$$
\begin{aligned}
& f^{\mathrm{soc}}(-z) \\
& =f\left(\lambda_{1}(-z)\right) u_{-z}^{1}+f\left(\lambda_{2}(-z)\right) u_{-z}^{2} \\
& =f\left(-\lambda_{2}(z)\right) u_{z}^{2}+f\left(-\lambda_{1}(z)\right) u_{z}^{1} \\
& = \begin{cases}-f\left(\lambda_{2}(z)\right) u_{z}^{2}-f\left(\lambda_{1}(z)\right) u_{z}^{1} & \text { if } f \text { is odd, } \\
f\left(\lambda_{2}(z)\right) u_{z}^{2}+f\left(\lambda_{1}(z)\right) u_{z}^{1} & \text { if } f \text { is even, }\end{cases} \\
& = \begin{cases}-f^{\text {soc }}(z) & \text { if } f \text { is odd } \\
f^{\text {soc }}(z) & \text { if } f \text { is even. }\end{cases} \\
& \text { “ } \Rightarrow \text { " For } \tau \in \mathbb{R} \text {, we have } \\
& \begin{aligned}
f(-\tau) & =\left\langle f^{\mathscr{L}_{\theta}}(-\tau e), e\right\rangle \\
& = \begin{cases}\left\langle-f^{\mathscr{L}_{\theta}}(\tau e), e\right\rangle=-f(\tau) & \text { if } f^{\mathscr{L}_{\theta}} \text { is odd, } \\
\left\langle f^{\mathscr{L}_{\theta}}(\tau e), e\right\rangle=f(\tau) & \text { if } f^{\mathscr{L}_{\theta}} \text { is even. }\end{cases}
\end{aligned}
\end{aligned}
$$

The below example illustrates that the above relationship fails to hold for $f^{\mathscr{L}_{\theta}}$ and $f$.

Example 22. Let $f^{\mathscr{L}_{\theta}}$ be given as in (4) with $\tan \theta=2(\theta=$ $\left.\tan ^{-1} 2\right)$. Then,

(a) $f(t)=t^{3}$ is an odd function, but $f^{\mathscr{L}_{\theta}}$ is not an odd function at $z=(1,1)$;

(b) $f(t)=t^{2}$ is an even function, but $f^{\mathscr{L}_{\theta}}$ is not an even function at $z=(1,1)$.

For $f(t)=t^{3}$, it is clear that $f(t)=t^{3}$ is an odd function. Nonetheless, we verify that

$$
f^{\mathscr{L}_{\theta}}(z)=\frac{1}{20}(110,215), \quad f^{\mathscr{L}_{\theta}}(-z)=\frac{1}{20}(-50,-35),
$$

which says $-f^{\mathscr{L}_{\theta}}(z) \neq f^{\mathscr{L}_{\theta}}(-z)$. Thus, $f^{\mathscr{L}_{\theta}}$ is not an odd function at $z=(1,1)$.
Similarly, for $f(t)=t^{2}$ which is an even function, we compute

$$
f^{\mathscr{L}_{\theta}}(z)=\frac{1}{10}(20,35), \quad f^{\mathscr{L}_{\theta}}(-z)=\frac{1}{10}(20,5) .
$$

Hence, $f^{\mathscr{L}_{\theta}}(-z) \neq f^{\mathscr{L}_{\theta}}(z)$ which says $f^{\mathscr{L}_{\theta}}$ is not an even function at $z=(1,1)$.

Finally, let us discuss the relationship between circular cone and the (nonsymmetric) matrix cone introduced in $[17,22]$, where the authors study the epigraph of six different matrix norms, such as the Frobenius norm, the $l_{\infty}$ norm, $l_{1}$ norm, the spectral or the operator norm, the nuclear norm, and the Ky Fan $k$-norm. If we regard a matrix as a highdimensional vector, then the circular cone is equivalent to the matrix cone with Frobenius norm. More precisely, denote

$$
K_{m, n}^{\epsilon}:=\left\{(t, X) \in \mathbb{R} \times \mathbb{R}^{m \times n} \mid \epsilon^{-1} t \geq\|X\|_{F}\right\},
$$

where $\|X\|_{F}$ denotes the Frobenius norm of $X$; that is, $\|X\|_{F}:=$ $\left(\sum_{i=1}^{m} \sum_{j=1}^{n}\left|X_{i j}\right|^{2}\right)^{1 / 2}$. Notice that the circular cone $\mathscr{L}_{\theta}$ can be equivalently written as

$$
\mathscr{L}_{\theta}=\left\{\left(x_{1}, x_{2}\right) \in \mathbb{R} \times \mathbb{R}^{n-1} \mid x_{1} \tan \theta \geq\left\|x_{2}\right\|\right\} .
$$

Let $t=x_{1}, \epsilon=\operatorname{ctan} \theta$ and $X=\operatorname{diag}\left(x_{2}\right)$ where diag denotes the diagonal matrix; then $K_{n-1,1}^{\mathrm{ctan} \theta}$ reduces to $\mathscr{L}_{\theta}$. Conversely, the matrix cone $K_{m, n}^{\epsilon}$ can be also regarded as a circular cone with

$$
\begin{gathered}
\tan \theta=\epsilon^{-1}, \quad x_{1}=t, \\
x_{2}=\left(X_{11}, X_{12}, \ldots, X_{1 n}, \ldots, X_{i 1}, \ldots, X_{i n}, \ldots, X_{m 1},\right. \\
\left.\ldots, X_{m n}\right)^{T} \in \mathbb{R}^{m n} .
\end{gathered}
$$

Therefore, $K_{m, n}^{\epsilon}$ is a $(m n+1)$-dimensional circular cone.

In addition, we know for a vector $y \in \mathbb{R}^{n}$ (regarding as a matrix in $\mathbb{R}^{n \times 1}$ ) the singular value decomposition is

$$
y=U \Sigma V^{T}
$$

where $V=1 \in \mathbb{R}, \Sigma=(\|y\|, 0, \ldots, 0)^{T} \in \mathbb{R}^{n \times 1}$, and $U=\left[U_{1}, \ldots, U_{n}\right] \in \mathbb{R}^{n \times n}$ with $U_{1}=y /\|y\|$ and $U_{2}, \ldots, U_{n}$ are arbitrary orthonormal vectors orthogonal to $y$. It indicates that the singular value of $y$ is $\|y\|$ and 0 with multiplicity $n-1$. Hence the spectral/operator norm (largest singular value), the Ky Fan $k$-norm (the sum of $k$-largest singular value), or the nuclear norm (the sum of the singular values) are all $\|y\|$. This means that $\mathscr{L}_{\theta}$ is a special case of the matrix cone studied in $[17,22]$, where the properties of spectral operator are studied, such as well-definiteness, the directional differentiability, the Fréchet differentiability, the locally Lipschitz continuity, the $\rho$-order $B$-differentiability $(0<\rho \leq 1)$, the $\rho$-order G-semismooth $(0<\rho \leq 1)$, and the characterization of Clarke's generalized Jacobian. In this paper, by using the special structure of circular cone, we mainly establish the $B$-subdifferential (the approach we considered here is more directly and depended on the special structure of circular cone), the directional differentiability in the Hadamard sense, and the $\rho$-order semismooth for $\rho>1$. 


\section{Conflict of Interests}

The authors declare that there is no conflict of interests regarding the publication of this paper.

\section{Acknowledgments}

The authors are gratefully indebted to Professor Defeng Sun for drawing their attention to the relationship between circular cone and matrix cone introduced in $[17,22]$. In particular, the characterization of $B$-subdifferential $\partial_{B}\left(f^{\mathscr{L}_{\theta}}\right)$ given in Theorem 13 is inspired by the analysis technique used in [17, Chapter 3]. Jinchuan Zhou's work is supported by the National Natural Science Foundation of China (11101248, 11271233), Shandong Province Natural Science Foundation (ZR2010AQ026, ZR2012AM016), and Young Teacher Support Program of Shandong University of Technology. Jein-Shan Chen's work is supported by Ministry of Science and Technology, Taiwan.

\section{References}

[1] Y.-L. Chang, C.-Y. Yang, and J.-S. Chen, "Smooth and nonsmooth analyses of vector-valued functions associated with circular cones," Nonlinear Analysis. Theory, Methods \& Applications Series A: Theory and Methods, vol. 85, pp. 160-173, 2013.

[2] J.-C. Zhou and J.-S. Chen, "Properties of circular cone and spectral factorization associated with circular cone," Journal of Nonlinear and Convex Analysis, vol. 14, no. 4, pp. 807-816, 2013.

[3] J.-S. Chen, "Alternative proofs for some results of vectorvalued functions associated with second-order cone," Journal of Nonlinear and Convex Analysis, vol. 6, no. 2, pp. 297-325, 2005.

[4] J.-S. Chen, "The convex and monotone functions associated with second-order cone," Optimization. A Journal of Mathematical Programming and Operations Research, vol. 55, no. 4, pp. 363-385, 2006.

[5] J.-S. Chen, X. Chen, and P. Tseng, "Analysis of nonsmooth vector-valued functions associated with second-order cones," Mathematical Programming. A Publication of the Mathematical Programming Society B, vol. 101, no. 1, pp. 95-117, 2004.

[6] X. Chen, H.-D. Qi, and P. Tseng, "Analysis of nonsmooth symmetric-matrix-valued functions with applications to semidefinite complementarity problems," SIAM Journal on Optimization, vol. 13, no. 4, pp. 960-985, 2003.

[7] D. F. Sun and J. Sun, "Semismooth matrix-valued functions," Mathematics of Operations Research, vol. 27, no. 1, pp. 150-169, 2002.

[8] Y.-L. Chang and J.-S. Chen, "The Hölder continuity of vectorvalued function associated with second-order cone," Pacific Journal of Optimization, vol. 8, no. 1, pp. 135-141, 2012.

[9] T. P. Wihler, "On the Hölder continuity of matrix functions for normal matrices," JIPAM. Journal of Inequalities in Pure and Applied Mathematics, vol. 10, no. 4, pp. 1-5, 2009.

[10] L. Q. Qi, "Convergence analysis of some algorithms for solving nonsmooth equations," Mathematics of Operations Research, vol. 18, no. 1, pp. 227-244, 1993.

[11] H.-D. Qi, "A semismooth Newton method for the nearest Euclidean distance matrix problem," SIAM Journal on Matrix Analysis and Applications, vol. 34, no. 1, pp. 67-93, 2013.

[12] L. Q. Qi and J. Sun, "A nonsmooth version of Newton's method," Mathematical Programming A, vol. 58, no. 3, pp. 353-367, 1993.
[13] D. F. Sun and J. Sun, "Strong semismoothness of eigenvalues of symmetric matrices and its application to inverse eigenvalue problems," SIAM Journal on Numerical Analysis, vol. 40, no. 6, pp. 2352-2367, 2002.

[14] D. F. Sun and J. Sun, "Strong semismoothness of the FischerBurmeister SDC and SOC complementarity functions," Mathematical Programming. A Publication of the Mathematical Programming Society A, vol. 103, no. 3, pp. 575-581, 2005.

[15] Y. N. Wang and N. H. Xiu, "Strong semismoothness of projection onto slices of second-order cone," Journal of Optimization Theory and Applications, vol. 150, no. 3, pp. 599-614, 2011.

[16] F. Facchinei and J.-S. Pang, Finite Dimensional Variational Inequalities and Complementarity Problems, Springer, New York, NY, USA, 2003.

[17] C. Ding, An introduction to a class of matrix optimization problems [Ph.D. thesis], National University of Singapore, Singapore, 2012.

[18] J.-P. Aubin and H. Frankowska, Set-Valued Analysis, Springer, New York, NY, USA, 2009.

[19] R. T. Rockafellar and R. J. Wets, Variational Analysis, Springer, New York, NY, USA, 1998.

[20] A. Shapiro, "On concepts of directional differentiability," Journal of Optimization Theory and Applications, vol. 66, no. 3, pp. 477487, 1990.

[21] J.-C. Zhou, J.-S. Chen, and H.-F. Hung, "Circular cone convexity and some inequalities associated with circular cones," Journal of Inequalities and Applications, vol. 2013, p. 571, 2013.

[22] C. Ding, D. F. Sun, and K.-C. Toh, "An introduction to a class of matrix cone programming," Mathematical Programming. A Publication of the Mathematical Programming Society A, vol. 144, no. 1-2, pp. 141-179, 2014. 


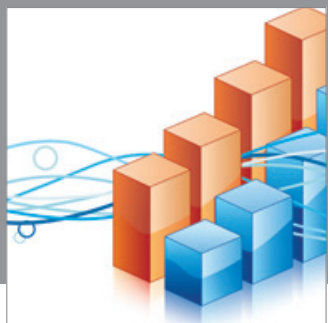

Advances in

Operations Research

mansans

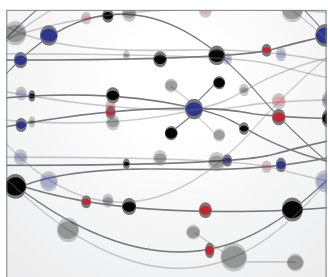

The Scientific World Journal
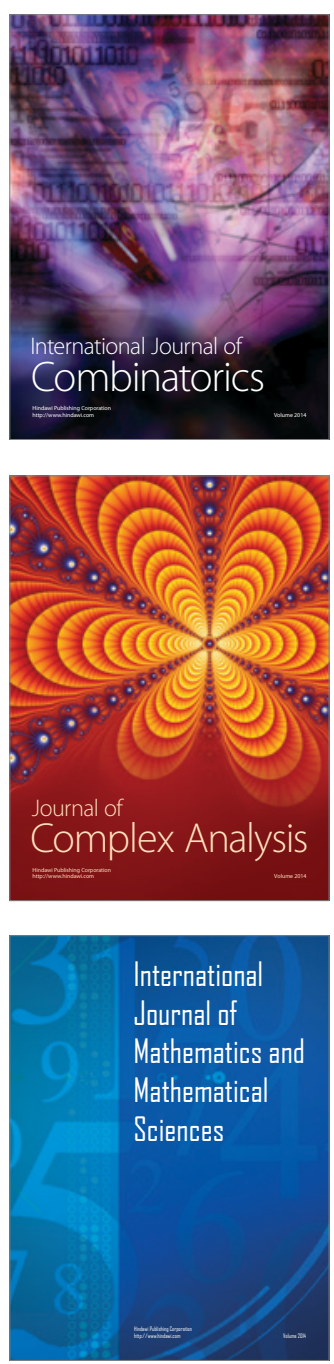
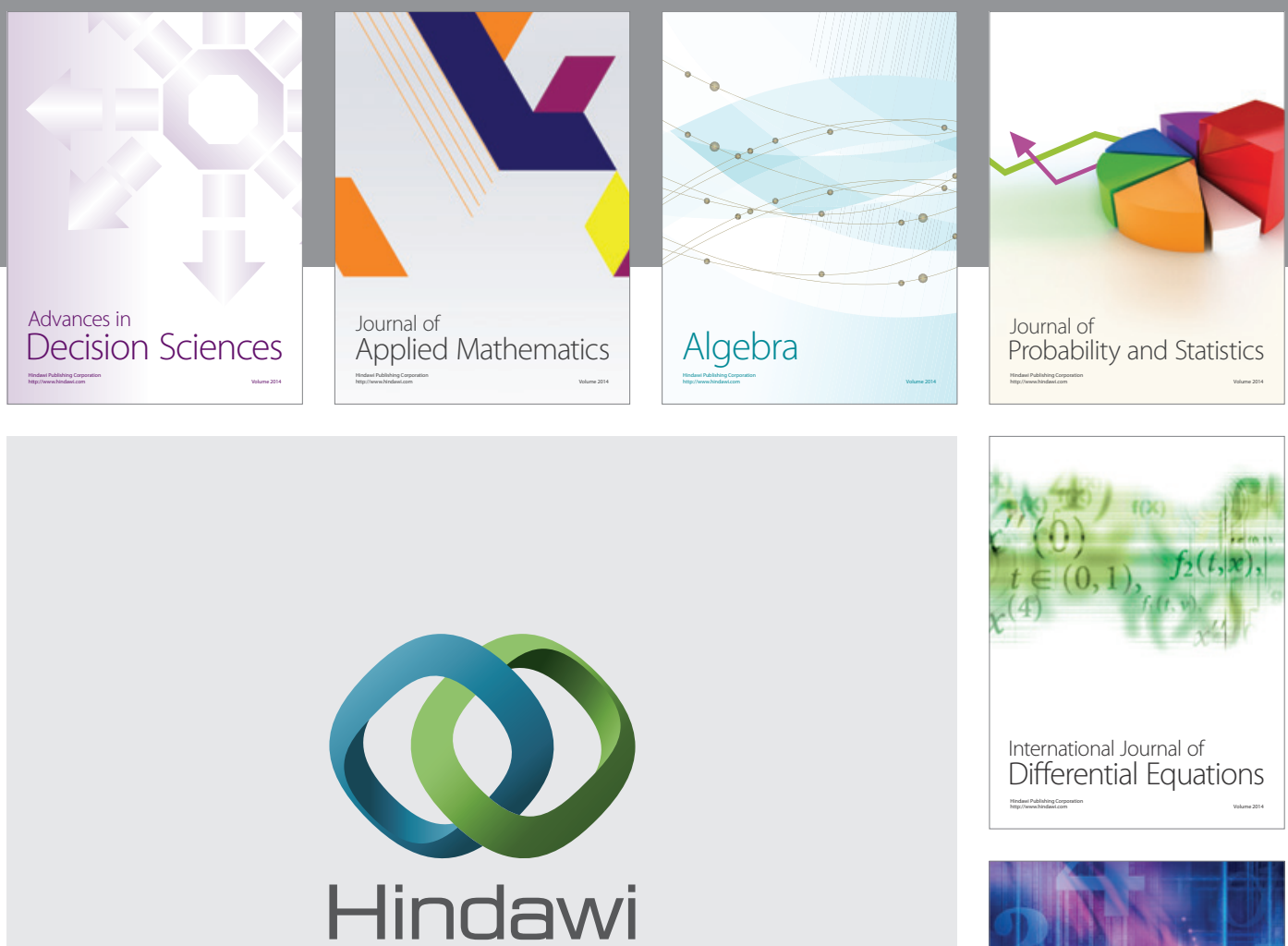

Submit your manuscripts at http://www.hindawi.com
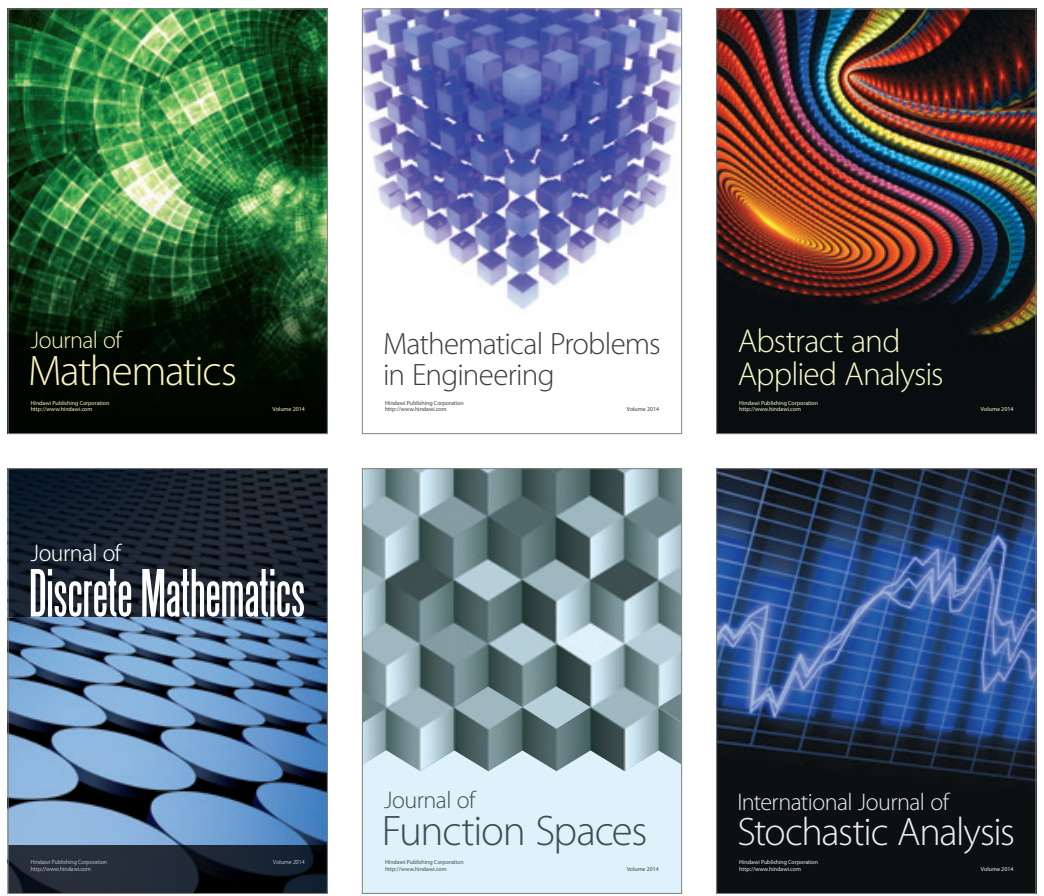

Journal of

Function Spaces

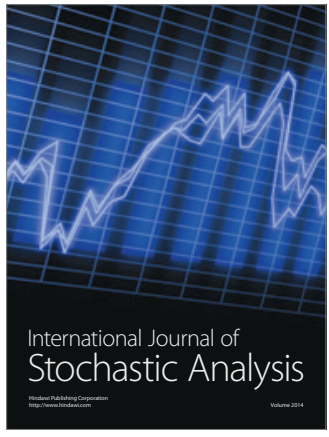

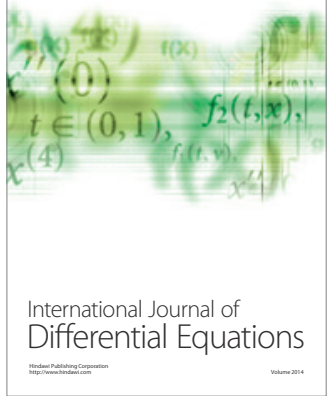
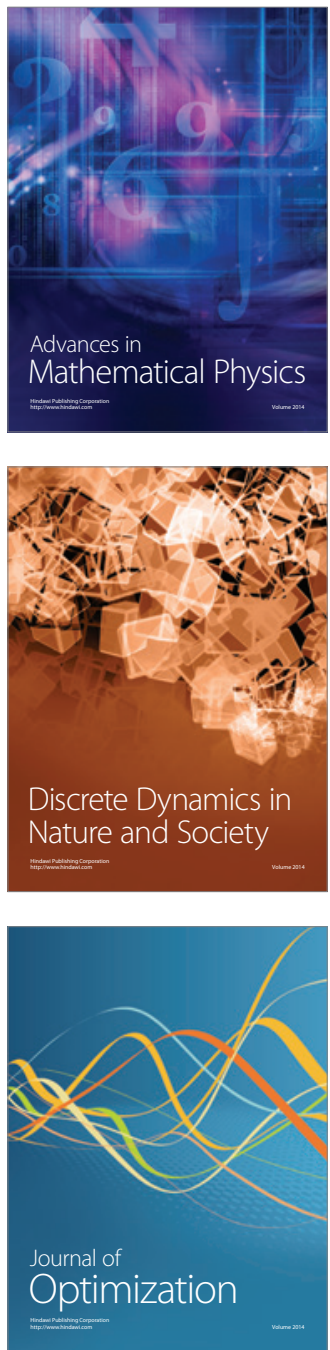